\title{
THE USE OF BIOLOGICAL TESTS FOR THE EXAMINATION OF PESTICIDES
}

\author{
ALFRED KAMIŃSKI \\ Medical Academy, Chair of Medical Biology, 90-647 Łódź, Plac 9-Maja 1, \\ Poland
}

\begin{abstract}
The increasing input of chemicals to the environment points to the need to control the input of pesticides. This requires a detailed analysis, not only of pesticide efficiency, but also of ecological response of the environment to a pesticide, before it is introduced for common application. Such an analysis should cover: (1) spectrum of species sensibility, (2) results of qualitative and quantitative bioassay analyses, (3) community response to the pesticide contamination, (4) neutralization, toleration and adaptation abilities of ecosystems, (5) share of a given pesticide in total toxity caused by the chemical contamination, (6) ecological predictions of environment contamination with pesticides. All these features of a pesticide can be expressed in numbers or by graphs with numerical axes. The paper presents a project, based on personal experiments, of an application of multi-assay bioindicatory analysis for the ecological evaluation of pesticides already used or being introduced for use. The experimental animals were: Asellus, Lumbriculus, Cloeon, Daphnia, Planorbarius, Lebistes. They were used as bioindicators of phospho-organic insecticide applied in 0.01-0.001 p.p.m. concentrations. The basis for bioindication of poisons in environments, and their characteristics in relation to the above six points were accomplished with the help of bioassay and the application of models of aquatic and terrestrial ecosystems.
\end{abstract}

\section{GENERAL METHODOLOGICAL PRINCIPLES}

The use of living organisms for studies of pesticides has a rather long history ${ }^{1,2,3}$. The main objective of these tests, which have been conducted on many species and with different techniques, is to estimate:

(1) Practical value of pesticides,

(2) Mechanism of action,

(3) Damage to human health,

(4) Methods of detection and quantitative evaluation.

The increasing contamination of the environment with chemicals and a threat to the normal functioning of biocoenoses made it necessary to extend the above-mentioned range of investigations so as to provide information on the ecological consequences of the use of pesticides in the quantities needed for plant protection. The rate of treatment with pesticides is limited by health factors and by the need to protect the environment against excessive contamination. 


\section{ALFRED KAMIŃSKI}

Ecological screening should involve determination of the major environmental effects of a pesticide and in particular:

(1) The biotaxonomic spectrum of toxic activity (BSTA), i.e. threshold concentrations contaminating the bionts being representative of polluted biocoenoses.

(2) Response of ecosystem structure and function to pollution,

(3) Persistence of pesticides in the environment,

(4) Effect of pollution with pesticides on the cumulative toxicity of all the coexisting chemical pollutions in the environment.

In all, the obtained information should extend the scientific criteria of pesticide evaluation and facilitate their qualitative optimization and rational application.

Long-term field and laboratory experiments are needed in ecological studies. The other disciplines involved here, such as biology, medicine, physicochemistry and agricultural technology, provide results which are frequently difficult to coordinate and interpret objectively.

Because of the complexity of the environmental (ecological) toxicology of applied poisons it is difficult to carry out such investigations in one laboratory.

Rapid evaluation of the properties of a pesticide (particularly one which is being introduced on to the market) requires the use of environmentaltoxicological techniques which should be as simple as possible.

The objective of this report is to present such a simplified system based exclusively on biological test analysis (TA).

TA is a research process in which a test biont (TB) or an ecological test system (ETS), for instance, a laboratory model of the ecosystem, performs the role of a double indicator in relation to the pesticides, which permits the worker:

(1) to detect and quantitatively estimate biologically active substances,

(2) to obtain a complete toxic-dynamic characteristic (TDC).

The primary reason for the use of TA for both purposes was to preserve as far as possible the structure of the polluted material (water, soil, air, tissues) as a result of elimination of complex, physical and chemical treatments.

The second idea was to introduce as a subject of analysis the quantitative determination of the actual toxicity of pesticides under existing environmental conditions. This actual toxicity is the net value of all additive, synergistic and antagonistic interactions in environments polluted with pesticides.

The third idea was to adjust the technique so that it could be used in conjunction with any chemicophysical analysis (CPA) and not in competition with it. This adjustment consists of an appropriate stimulation of the sensitivity and selectivity of TA. For this purpose it was necessary to broaden the TA method by the introduction of new and particularly sensitive test bionts (STB) and to develop new analytical criteria for the identification of pesticides. It was also necessary to create an artificial (control) analytical environment common to all the TB, and to unify the techniques of investigations in different natural environments.

The fourth idea was to minimize and speed up the process of analysis so that it could keep pace with the environmental-toxic processes stimulated by 
pesticides, without inhibiting them artificially, whether they were instantaneous or long-term.

The fifth idea was a maximum utilization of one TA conducted simultaneously for many TB species to obtain the greatest possible quantity of data characterizing the toxic-dynamic properties of a pesticide.

\section{Differences between the TA and CPA methods}

Qualitative identification of a pesticide with the TA method consists of the assessment of a set of symptons of the test response (TR) on TB (or ETS) that will be discussed below. As is well known, the quantitative estimation of a toxicant consists of the assessment of a threshold concentration $\left(C_{50}\right)$, which produces a TR in 50 per cent of the TB population.

Thus, TA indicates the effect of biologically active substances, and on the basis of replicability of this direct effect a conclusion may be drawn as to the nature and concentration of the active poison. It therefore provides direct information to the toxicologist or to the specialist applying pesticides in practice.

CPA, by comparison, is based on measurements of chemical reactions and physical processes not related to the actual biological activity of the analysed substance. From the point of view of environmental (ecological) toxicology CPA provides information which may have little direct bearing on the natural environment under study. This is particularly so if factors not detected by CPA act to inhibit or stimulate the effects of a pesticide. Such a disjunction may also occur if the natural structure of a substance is broken down during its preparation for CPA.

Thus, TA measures the results of direct effects under conditions closely approximating to the natural environment. It also measures the biological effects of the activity of pesticides under replicable artificial conditions created by the investigator. But these artificial conditions must always be within the tolerance limit of TB. which can always be measured precisely in control systems. Therefore, the purity of the TB reagent is analogous to the purity of a chemical reagent or to the efficiency of a measuring instrument. This purity is determined by the limit of tolerance of the analysis to side factors (factors which are present but not analysed) which should not disturb the physiological homeostasis (condition) of TB. The role of the energy potential of homeostasis (EPH) for the practical application of TA will be analysed in the section concerned with methods. But it should be noted here that a TB particularly sensitive to pesticides, i.e. STB, allows the TA to be carried out in such a short time that many side factors, including other poisons, will not be able to disturb either EPH or the specificity of TR to a definite pesticide.

With TA one can determine not only particular pesticides but also the cumulative toxicity of the environment (water, soil, air, biomass) contaminated with chemicals. And in that case TA cannot be replaced by any other method.

Quantitative biochemical analysis (BCA) of organophosphoric pesticides, based for instance on the acyl choline matrix ${ }^{4,5,6}$ and which is in some way intermediate between CPA and TA, is characterized by a rather high sensitivity but it is not specific for particular toxicants and it is sensitive to the side factors. Matrix (receptor of a pesticide) in this method is 'naked'. not being protected by the cover provided by the TB biomass in each TA. Due to the 


\section{ALFRED KAMIŃSKI}

homeostatic nature of TB and the readily visible response of such an organism to pesticides, TA gains a valuable selectivity and loses none of the advantages of BCA.

Searching for new TB and, particularly, STB consists of the selection of small and common carriers of a reactive and specific receptor of the toxicants, which are surrounded by the most effectively functioning EPH cover. This cover should eliminate side factors, efficiently transmit the subject of analysis to the receptor, and produce a multisymptomatic TR, which is the basis of the functioning of TA. Methodologically more similar to TA are the tests of pesticides conducted on cellular organelles, on chloroplasts for instance ${ }^{7}$, involving biochemical measurements of the effect of the degree of inhibition of Hill's reaction but not taking into account the external symptoms of the TR, which could provide additional criteria of pesticide identification, besides the determination of a threshold concentration.

Similarly, the measurements of the inhibition by pesticides of enzyme activity in $\mathrm{TB}^{8}$ represent an interesting junction of the methodological principles of TA and BCA, which, however, do not specify toxic substances in the analytical sense. The above-mentioned techniques are of great importance for the idea of TA functioning although they have a rather theoretical and auxiliary character.

Certainly, the present short report cannot provide a more complete outline of the theoretical bases of TA nor ecosystemic test analysis of pesticides (ETA).

However, to characterize the 'ideology' of this technique, it should be stated that even the most detailed CPA cannot provide reliable evidence for the lack of toxic contamination of the environment if it is used separately, without any verification by TA, while TA used separately can also provide evidence of toxicity in relation to unidentified poisons present in the field (in a sample) independently of whether the appropriate CPA methods exist or not. Therefore, fully developed TA can provide a methodological basis for the functioning of a system of permanent bioindication (SPB) summing and evaluating the effects of chemical impact on the natural environment. SPB can act through STB permanently present in the environment as natural reactors (in situ). They play the role of micro-, macro- and megabioindicators of toxicity or effectiveness and ineffectiveness of treatments with chemicals.

The first step to create the STB at particular levels of organization of organic matter is a simple TA and ecological test analysis (ETA) leading to the selection of STB and to modelling of ecological processes of environmental contamination in the laboratory. Parallel and successfully developed CPA techniques for pesticides represent an indispensable research instrument for scientific interpretation of the processes occurring in environments polluted with chemicals. Combination of these techniques can provide the most useful results.

\section{TECHNIQUE OF TA REALIZATION}

It is impossible to present all technical details of TA in this short report and only the main methodological principles will be illustrated by the analysis of selected examples of pesticides and other toxic substances suffi- 
THE USE OF BIOLOGICAL TESTS FOR THE EXAMINATION OF PESTICIDES

ciently representative for the present pollution of the natural environment with chemicals.

\section{(1) Subject of TA}

(a) Standard toxicants $(S T)$

ST such as gamma-BHC (gamma-hexachlorocyclohexane), DDVP (2,2dichlorovinyl ester of dimethylphosphoric acid), Chlorofenvinphos (dichloro1-1/2,4 dichlorophenylvinyl ester of diethylphosphoric acid), PMPF (pinacoline ester of methylfluorphosphonic acid), which is an inhibitor of choline esterase killing vertebrates, and mercuric chloride $\left(\mathrm{HgCl}_{2}\right)$ were tested in the form of impregnates on pure starch. The method of complete TB was used according to the technique described below, in aquatic and standard terrestrial environments both being strictly replicable (ST formulaFigure 1).<smiles>CC(C)(C)OP(C)(=O)P</smiles><smiles>COP(=O)(OC)OC=CCl</smiles>

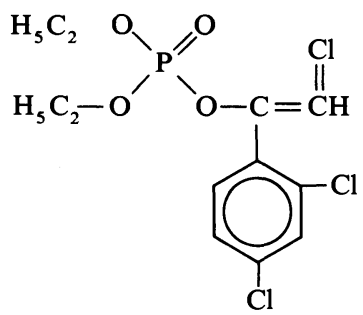<smiles>ClC1C(Cl)C(Cl)C(Cl)C(Cl)C1Cl</smiles>

Chlorofenvinfos (ChLVF)

Figure 1. Toxicants used as examples in TA

(b) Dilution of toxicants

All insecticide ST were first diluted in acetone at a concentration of $1 \mathrm{~g} / 100 \mathrm{ml}$; the highly toxic PMPF was similarly diluted in propylene glycol, and mercuric chloride in redistilled water. These concentrated solutions were used to prepare aqueous working fluids, the concentrations of which are indicated in the tables and figures. The series of concentrations of the working fluids, used to impregnate the starch matrix, forms a decreasing geometric progression with the ratio two.

(c) Introduction of toxicants to ecological test systems (ETS)

Aquatic ETS of a volume of $4 \mathrm{~L}$ were contaminated with 11 of natural water (from a culture of TB) containing an appropriate concentration of ST indicated in the tables. Terrestrial ETS were contaminated by the impregnation of soil with aqueous-acetone solutions of ST at the concentration of acetone tolerated by all TB. 


\section{ALFRED KAMIŃSKI}

(2) Test objects (Figures 2-8)

The number of TB in the present biological and toxicological investigations of pesticides is difficult to define $\mathrm{e}^{9,10}$. In fact, they are as numerous as the pest species of animals and plants treated with pesticides. Besides, the response to pesticides has been determined for many species of bionts subjected to treatment with pesticides in the field ${ }^{11,12,13}$.

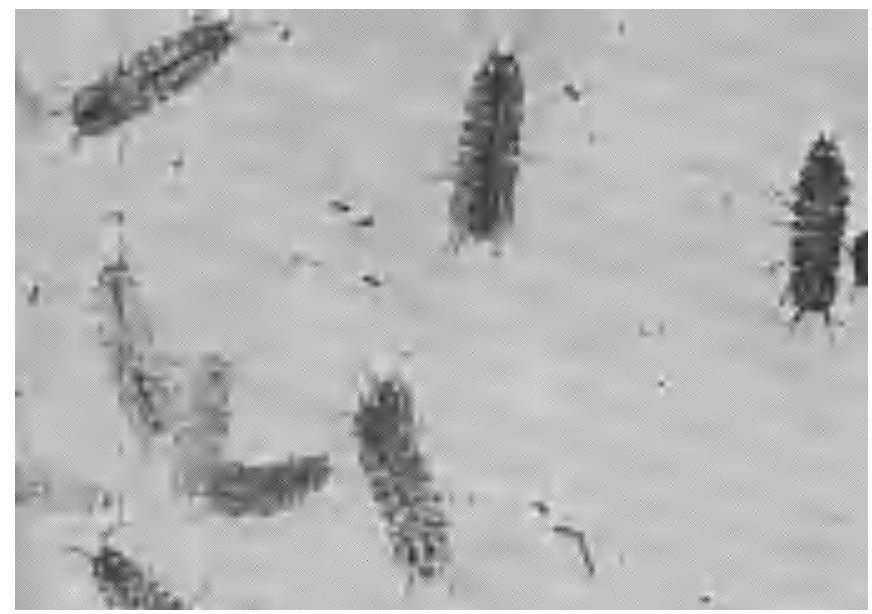

Figure 2. TB-Asellus aquaticus (Isopoda)

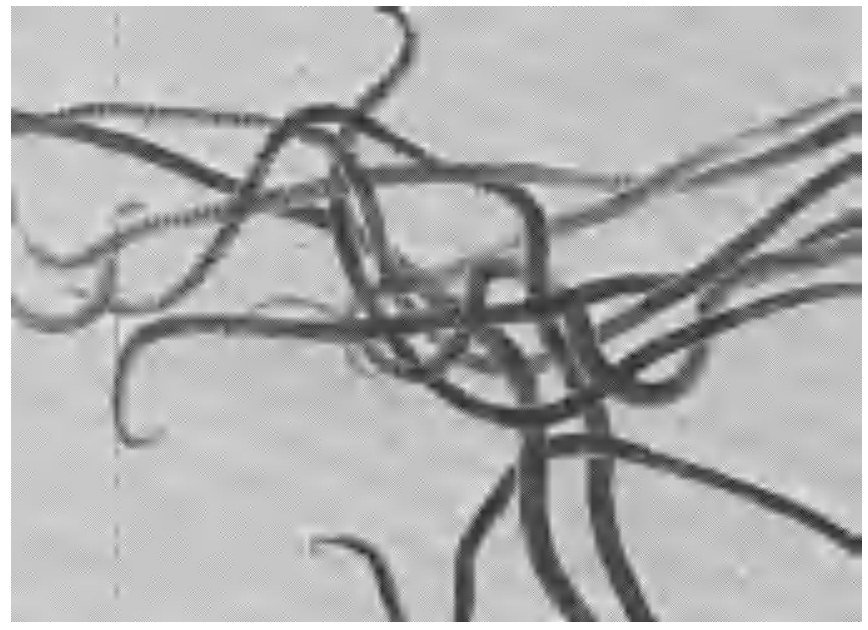

Figure 3. TB-Lumbriculus variegatus (Oligochaeta) 


\section{THE USE OF BIOLOGICAL TESTS FOR THE EXAMINATION OF PESTICIDES}

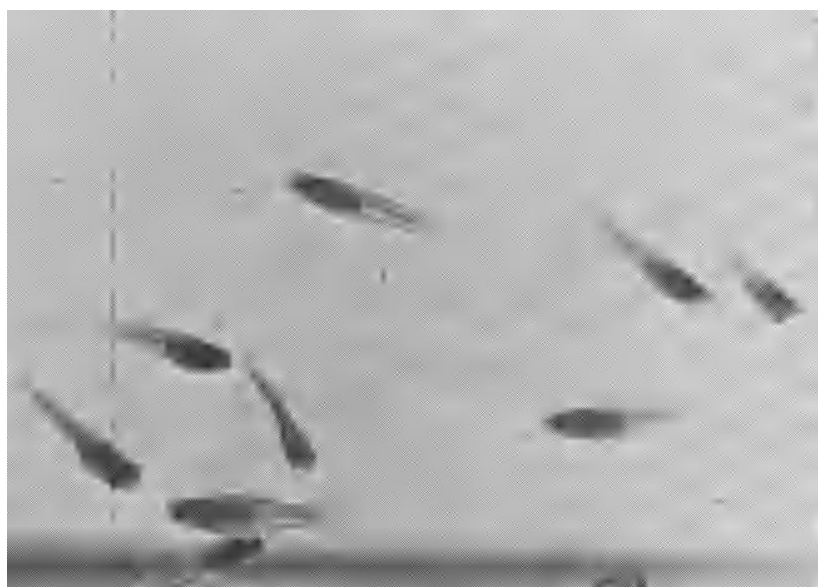

Figure 4. TB-Lebistes reticulatus

When selecting the TB for these experiments account was taken of the necessity for miniaturization and for economy of analyses, and also other criteria such as:

(1) Survival rate in small analytical containers,

(2) Mass occurrence in nature,

(3) Sensitivity to toxicants-frequently from $0.1-0.001$ p.p.m.

(4) Pronounced symptomatology of test response,

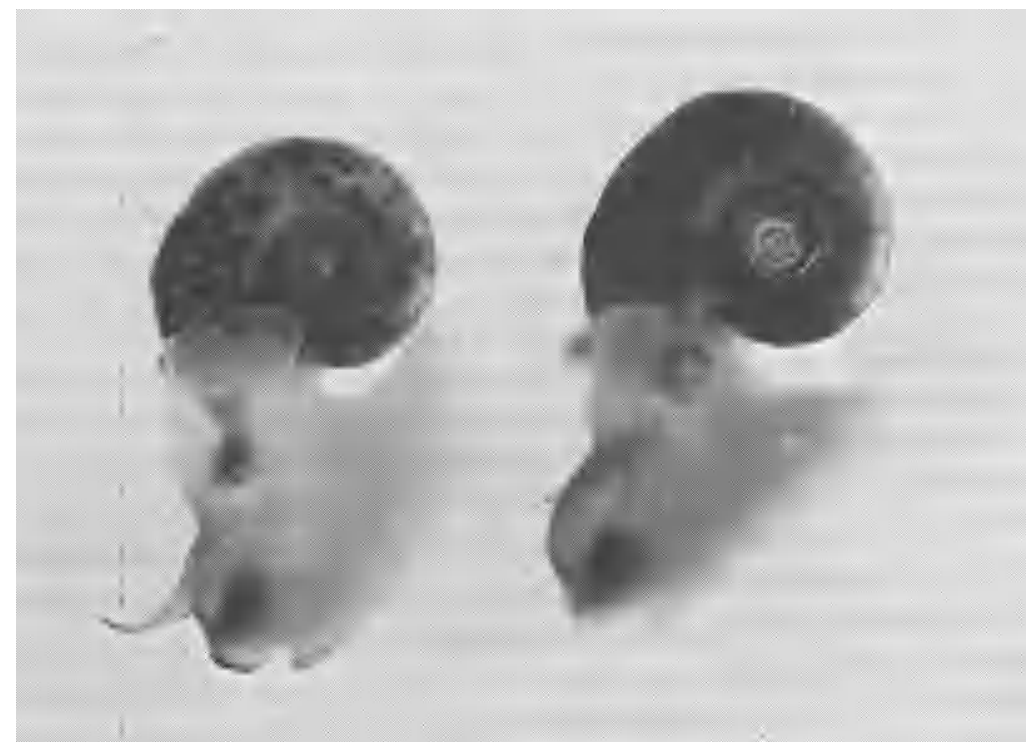

Figure 5.TB-Planorbarius corneus with TR symptons of DDVP 


\section{ALFRED KAMIŃSKI}

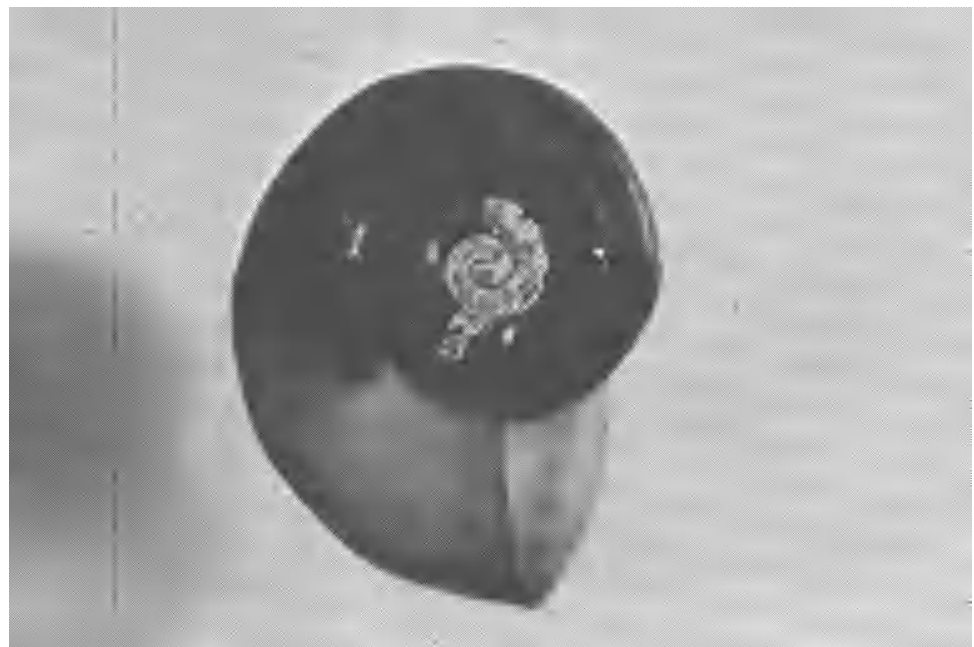

Figure 6. TB-Planorbarius corneus with TR symptons of $\mathrm{HgCl}_{2}$

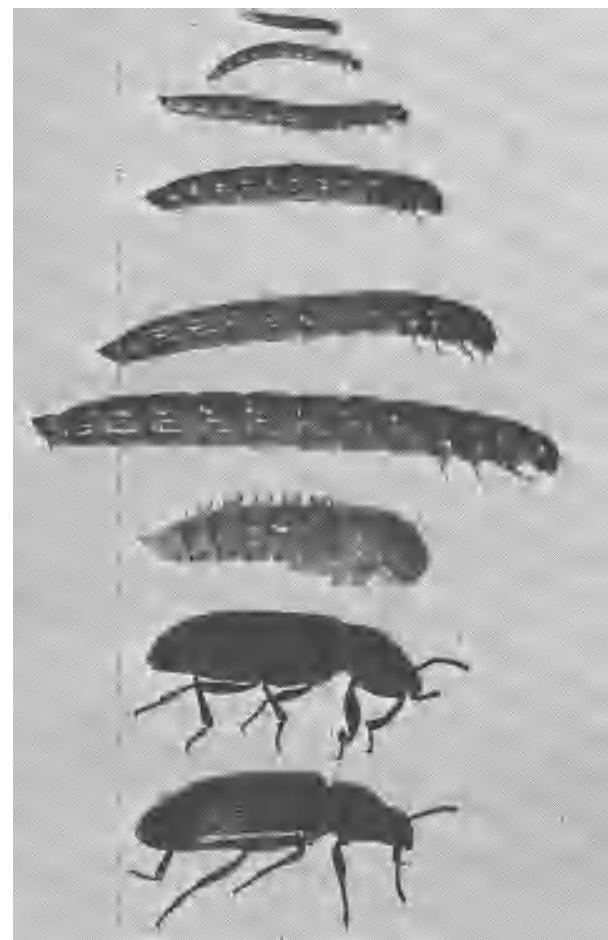

Figure 7. TB-Tenebrio molitor (development phases) 


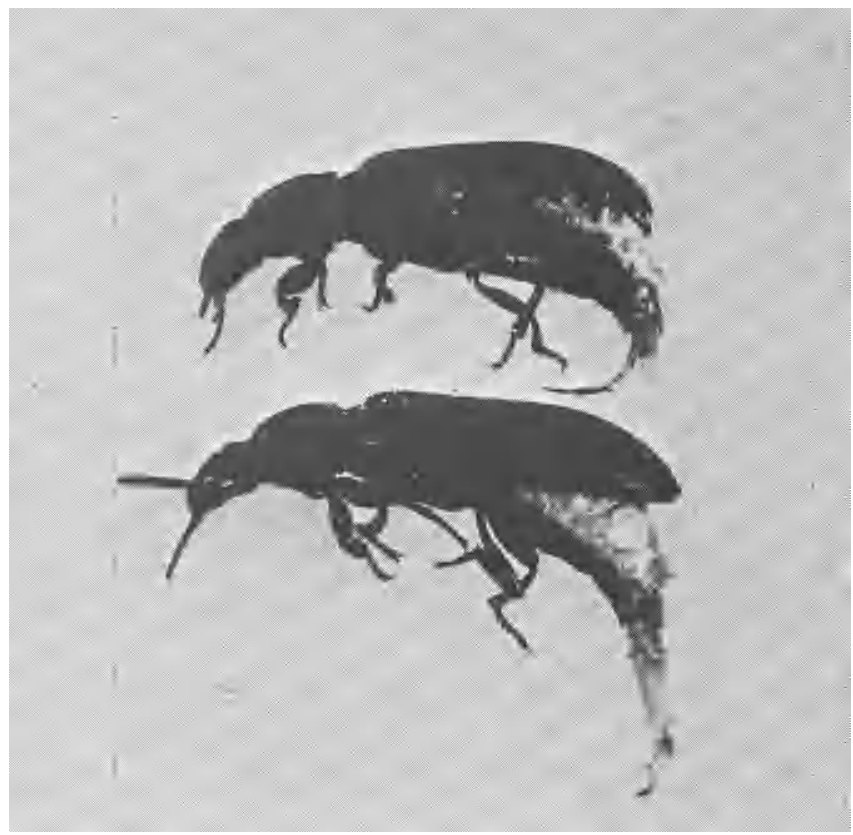

Figure 8. TB-Tenebrio molitor with TR symptoms of DDVP

(5) Facility of mass rearing in the laboratory,

(6) Longevity,

(7) High value of EPH defending reactive receptors of TB against side factors, i.e. pollutions under different environmental conditions,

(8) Ecological accordance of hydrobionts.

(9) Survival rate in a common standard (replicable) environment,

(10) Facility of transportation during scientific expeditions and also in hermetic containers.

The following species of hydrobionts were included in the TB system: from crustaceans-Asellus aquaticus(Isopoda) and Daphniamagna(Cladocera), from annelids-Lumbriculus variegatus (Oligochaeta), from fish-Lebistes reticulatus, from unicellular organisms-Spirostomum ambiguum (Ciliata).

Besides hydrobionts, one terrestrial species of insect, Tenebrio molitor, was selected. It has an average sensitivity to insecticides as TB determining the practical value of insecticides. Its important advantages are the normal survival in dry, loose, artificial environment and incapability to climb the glass walls of analytical containers.

Besides Daphnia magna (used in short-term analyses), the remaining objects, including unicellular organisms, are long-lived, which is of great importance to TA.

Selection of TB for analyses was conducted according to the principles presented in the table, where also their specific sensitivity to some toxicants is indicated in p.p.m. (Tables 1 and 2). 


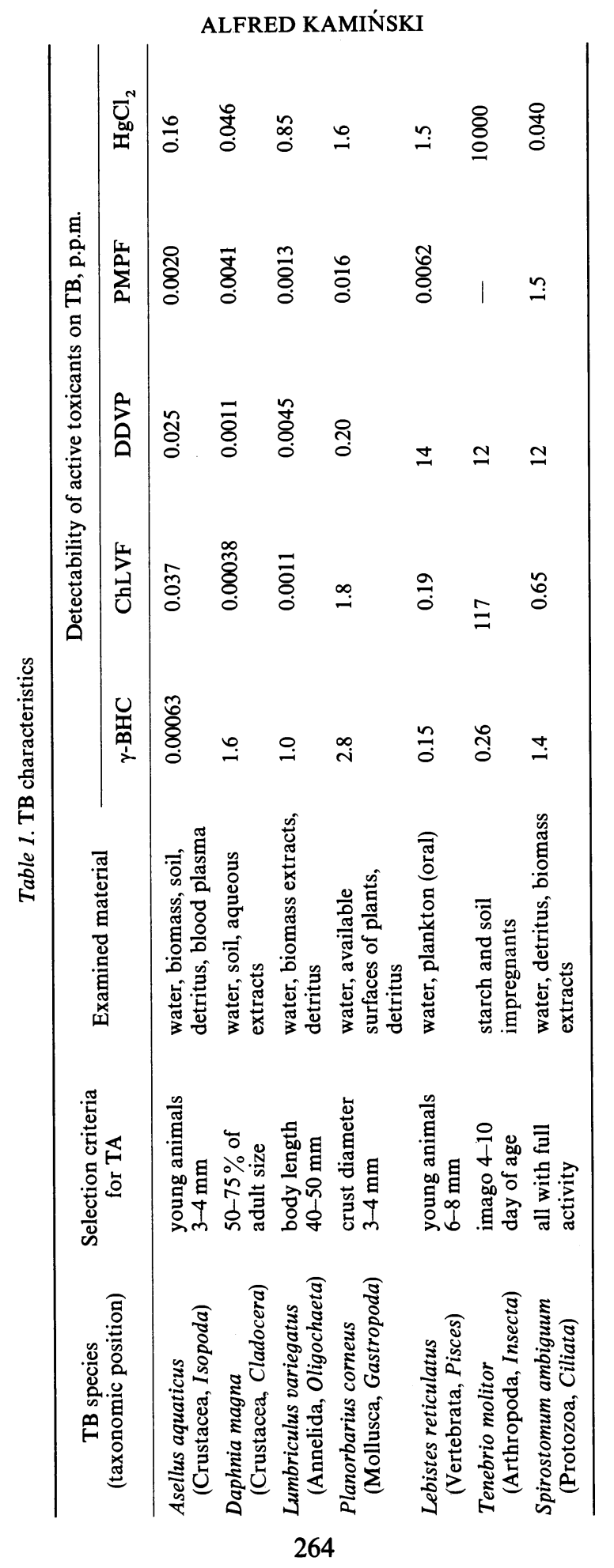


THE USE OF BIOLOGICAL TESTS FOR THE EXAMINATION OF PESTICIDES

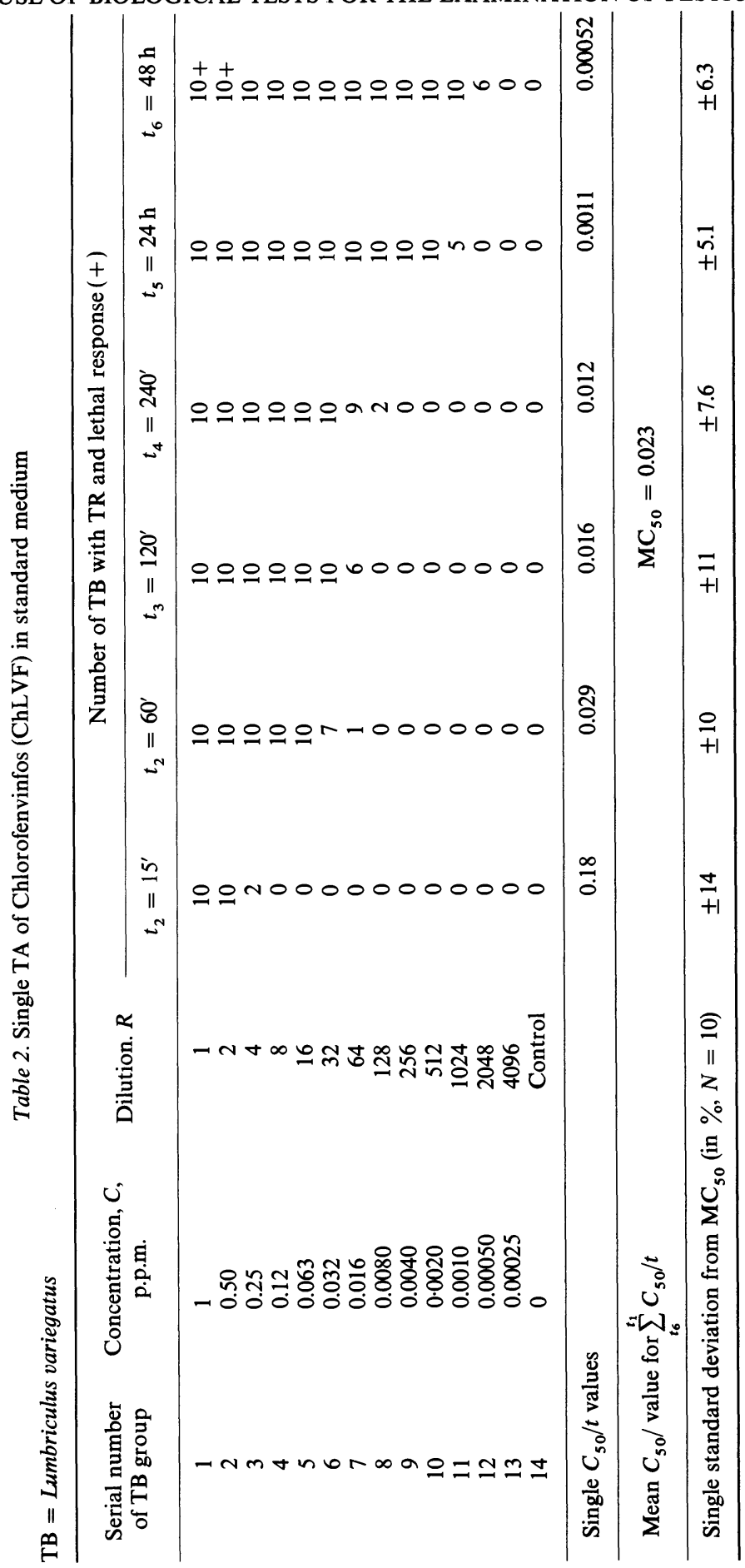


(3) Experimental environments (EE)

(a) Aquatic (AEE)

The mineral composition of the diluent common for the tested toxic substances (EE) was determined for all aquatic TB. It contained the following compounds, excluding water of crystallization: $\mathrm{NaCl} 1.00, \mathrm{KCl} 0.250, \mathrm{MgCl}_{2}$ $0.120, \mathrm{CaCl}_{2} 0.250, \mathrm{NaHPO}_{4} 0.00620, \mathrm{NaHCO}_{3} 0.125$, and redistilled water to 1000.0 .

This composition is the equivalent of Tyrode's fluid diluted eight times. For Spirostomum the described AEE was additionally diluted eight times with redistilled water prepared in a quartz apparatus without metallic connectors as this animal is sensitive to traces of metals above 0.01 p.p.m.

The quality and proportions of the concentration of particular components of $\mathrm{EE}$ correspond to the electrolytes of vertebrate blood. So, it is possible to use TB for the analysis of body fluids and tissues contaminated with insecticides.

Environmental-toxicological analyses are generally conducted in filtrated natural water coming from the TB cultures. Only when the effect of the natural water on the results of analysis should be determined, or a verdict evaluating the quality of a pesticide, comparable with that of other authors is needed, should EE be used together with an identical (international) standard of the tested substance.

\section{(b) Terrestrial (TEE)}

They represent systems in many respects similar to a fluid environment, despite their dryness. This is pure potato starch. It readily disperses toxicants through the impregnation and evaporation of solvent. Evaporation is either complete (acetone, ethanol, ether, benzene, cyclohexanone, water) or partial, as in the case of water. Due to such partial evaporation a very valuable model approximating moist soil with any proportions of minerals or other components of a natural biotop can be obtained.

Similarity between the terrestrial and aquatic EE results from the precise dispersal of a toxic substance due to impregnation, capillarity, mixing and movements of the particles of impregnant in the presence of mobile TB, which are adapted to a loose and dry environment as they are flour-dwelling organisms. It has been found that the replicability of the results of TA in impregnants and aqueous solution is identical ${ }^{14}$. Powder preparations can also be dispersed in the starch in dry form by grinding weighed portions.

\section{(c) Samples of natural environments (NE)}

The use of TA for the ecological studies of pesticides requires the determination of their residues (residual toxicity) in various materials and environments (soil, sediments, tissues, air). Generally, each material containing a toxic substance can be tested by TA through the direct contact with TB. Asellus and Lumbriculus live in and feed on plant tissues. Great concentration of plant material in a sample does not disturb the physiological condition of these TB. Animal tissues, blood and soil are also tested through direct contact or in homogeneous extracts diluted according to the technical principles of TA described below. Duration of the analysis of NE is limited only by a danger of the decay of test material. 


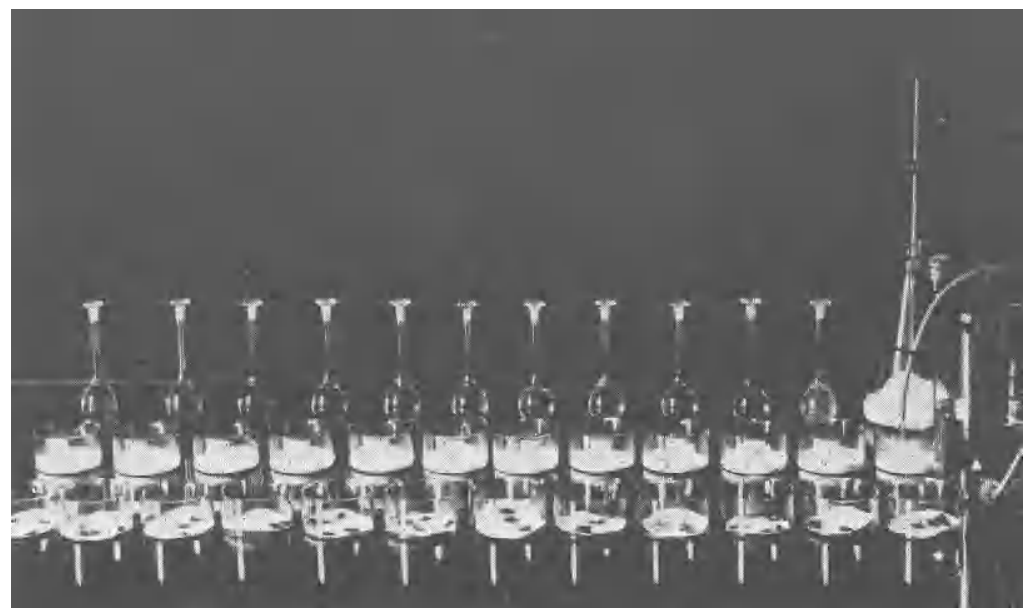

Figure 9. TA of insecticide dilution series in dry medium (potato starch), TB = Tenebrio molitor

In our experiments the residues of toxicants in aqueous-acetone extracts from whole animals such as mice and fish (without homogenization) were tested, and also the residues in fragments of organs by placing STB in direct contact with them. The content of the volatile vapours of insecticides, e.g. DDVP in the air, was directly determined through the exposure of a terrestrial TB in miniaturized aerodynamic or static chambers, and a parallel washing control and TA on hydrobionts (STB). Double bi-environmental TA of vapours and gases provides valuable results for many practical purposes such as biological examination of pesticides and sanitary control of the work conditions for the staff of chemical plants producing pesticides (Figures 9 and 10 ).

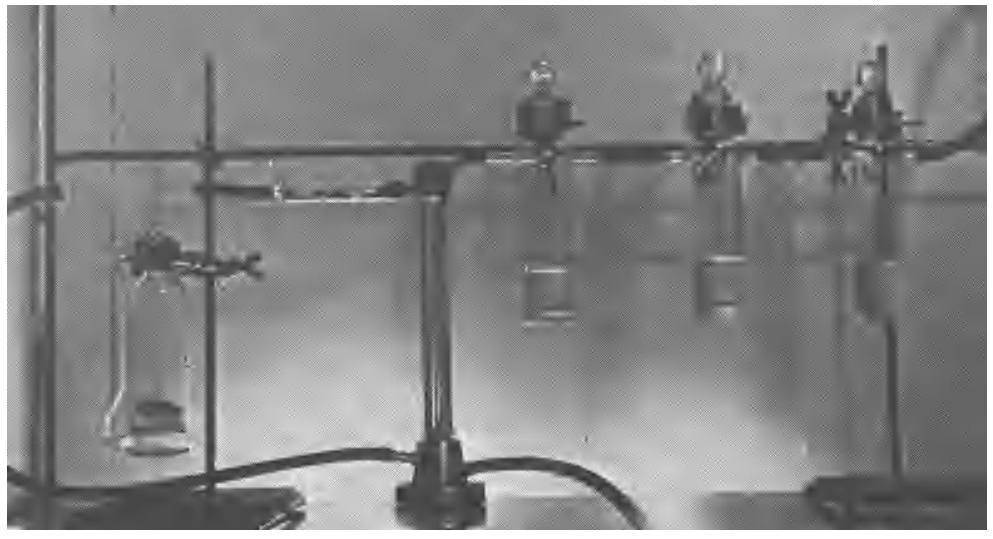

Figure 10. A microdynamic air chamber $(50 \mathrm{ml})$ with TB (Tenebrio), steam generator rotameter and three communicating washers for bi-environmental TA of insecticide steam 


\section{ALFRED KAMIŃSKI}

\section{(4) Test response (TR)}

Unlike the majority of the accepted methods of TA by which $\mathrm{LC}_{50}$ is determined, i.e. the concentrations of toxic substances producing lethal response (LR) of TB, in our experiments the symptoms of TR are analysed in vivo. TR is a replicable set of symptoms produced in response to the effect of a toxicant on TB. Lethal response has a supplementary importance as a specifying criterion. TR always occurs much earlier than LR and at concentrations $\left(C_{50}\right)$ many times lower (Table 2$)$.

Motor paralysis is a common and readily visible TR to insecticides in Asellus, Daphnia, Tenebrio and Lebistes. Additional symptoms are such as hypercinesia, which precedes TR, and the sequence of paralysing particular organs.

Organophosphoric inhibitors of choline esterase (IChE) primarily paralyse breathing (movements of the gill cover) and then the heart, which is readily visible through the integument of small TB. In crustaceans a prolonged agitation of branchial limbs is observed. It is different in the presence of IChE (acceleration) than in the presence of $\gamma$-BHC (discordance). Mercury paralyses respiratory movements (akinesis) in Asellus.

The results of TA presented in the tables and figures concern TR in Arthropoda, fish and Spirostomum; their motor activity and static reflexes are paralysed. Special attention should be paid to TR in Lumbriculus. This organism is characterized by permanent mobility, and it rapidly escapes in response to a touch stimulus. This response can be reversed in the presence of IChE at very low concentrations - the irritated TB falls in a tonic contraction (spiral kink) instead of escaping. This response is a result of the inhibition of acylcholine esterases, which are very active in this $\mathrm{TB}^{13}$, and it disappears within 3-16 days after the separation of TB from the poison. Reversibility of TR is specific for particular IChE and represents a criterion specifying them. High concentrations of IChE result in an irreversible response leading to LR.

The differences between $C_{50}$ and $\mathrm{LC}_{50}$ are also specific for particular toxicants. Detailed symptomatology of TR is the subject of a separate publication (in press) and exceeds the framework of this report. The differences between $C_{50}$ and $\mathrm{LC}_{50}$ also occur in relation to other TB.

\section{(5) Quantitative TA}

The methodological basis of TA is the assessment of the maximum dilution of a toxicant $\left(R_{50}\right)$ which can produce TR in 50 per cent of the TB population within a definite time of absorption, $t$. In analyses of standard insecticides, the concentration of the prepared solution is known and the assessment of $R_{50}$ provides direct information about $C_{50}$. When the material polluted with a toxicant of unknown concentration is analysed, only $R_{50}$ can be determined and this value can be used for the calculation of $C_{50}$ by comparing it with the standard.

The absolute concentration of a toxicant in the material can be calculated according to the simple formula

$$
C=\left(R_{\mathrm{a} 50} / R_{\mathrm{s} 50}\right) \cdot f
$$

where $C$ is the concentration of poison in the sample (p.p.m.), $R_{\mathrm{a} 50}$ is the 
threshold dilution of the analysed solution, $R_{\mathrm{s} 50}$ is the threshold dilution of the standard solution, and $f$ is the concentration of the standard toxic substance in the standard solution (p.p.m.).

To find the value of $R_{50}$, an analytical sequence of poison dilutions must be prepared according to an optimum quotient of the geometric series $(q)$. Generally, $q=2$ is used to find room in the limited number of containers for the whole set of analyses ranging from 100 to 0 per cent of responding TB at all time-moments when TR is recorded, i.e. for numerous $t$. For special purposes and to increase the accuracy of TA, $q$ ranging from 1.5 to 1.05 is used, e.g. for a precise estimation of pesticide quality, identification of poison or toxicological verdicts.

\section{(6) Calculation of $\boldsymbol{R}_{\mathbf{5 0}}$ and statistics}

$R_{50}$ can be calculated according to any interpolation probit statistic ${ }^{15}$. In our investigations Wiechnowski's method ${ }^{16,17}$ was used. This method involves calculation not interpolation and has an element of self-control. It needs a regular course of TB response in the analytical sequence, i.e. a response proportional to the dosage. This analysis may be modified by appropriate selection of the $q$ value and by high quality of TB. The confidence interval presented in the tables is determined by two standard deviations. After five replications of the analysis, the standard deviation does not exceed 8-15 per cent, except for Daphnia magna, which is in the interval of 12-17 per cent.

\section{(7) Parameter of time in TA}

The time-period $(t)$ needed to produce TR in TB in contact with a toxicant along the dilution gradient depends on its concentration and rate of absorption from the environment. TR was measured along the decreasing gradient of concentrations at time-intervals $t$ selected according to a geometric progression. High frequency of records at the beginning of the gradient is necessary because of a rapid increase in TR at high concentrations of the toxic substance.

Lower frequency of records at the end of the gradient (low concentrations) coincides with a slow increase in TR or with the lack of increase when a compensation point is reached between the resorption rate and TB tolerance to the threshold concentration of a toxic substance. In the presence of $\gamma$-BHC the response of Tenebrio disappears within the range of 1-2 dilutions because of the metabolic adaptation of TB (internal detoxification).

The time-moment when the increase in TB response along the dilution gradient is stopped represents an important parameter of the qualitative specification of a toxicant. It is designated by the symbol $t \mathrm{EA}$ and expressed in minutes. It characterizes the critical time-moment of effective absorption and gives the idea of lower or higher cumulation of the toxicant in TB, and also of the chemical persistence of this substance in the environment.

The duration of TA depends on the purpose of the analysis. It is determined not only by $t$ EA but also by the need for collecting additional information, such as lethal response (LR), which follows TR after the period characteristic for each toxicant. To estimate the biological activity of new insecticides by the 


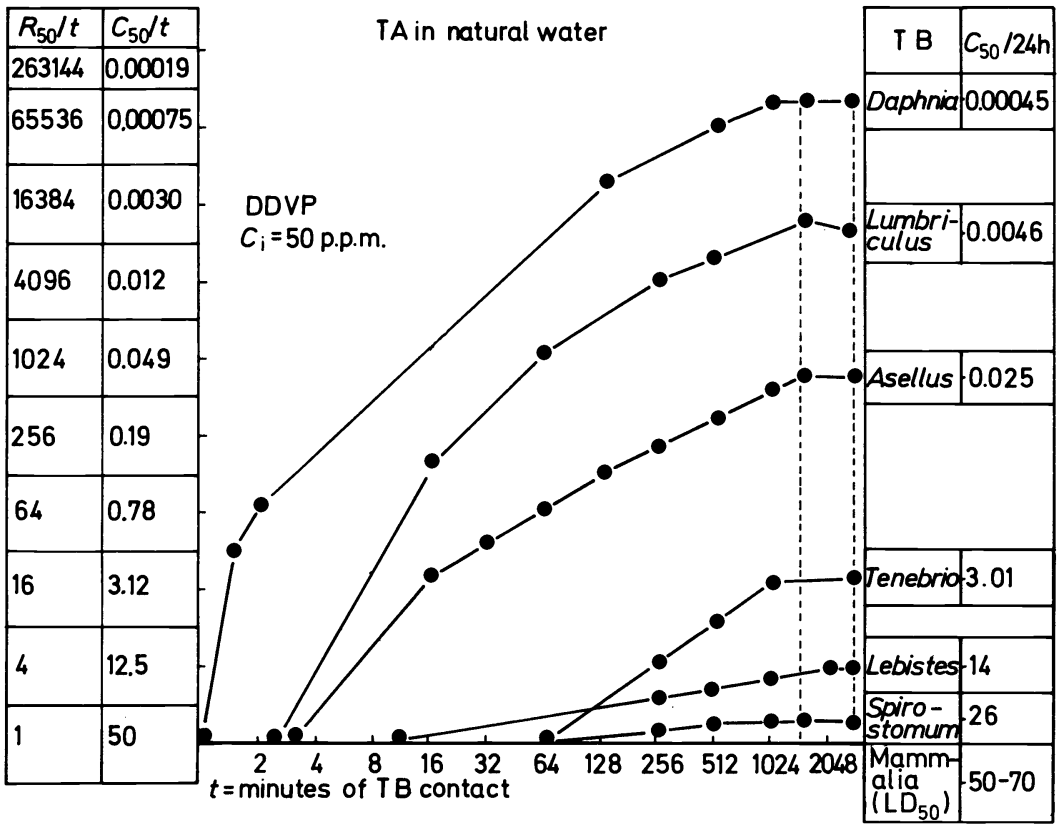

Figure 11. A multitest TA of DDVP in fresh (natural) water, the progress of TR in dilution series, $C_{50} / t$ values for Tenebrio from TA in dry medium, for Mammalia after parenteral application in $\mathrm{mg} / \mathrm{kg}=$ p.p.m.

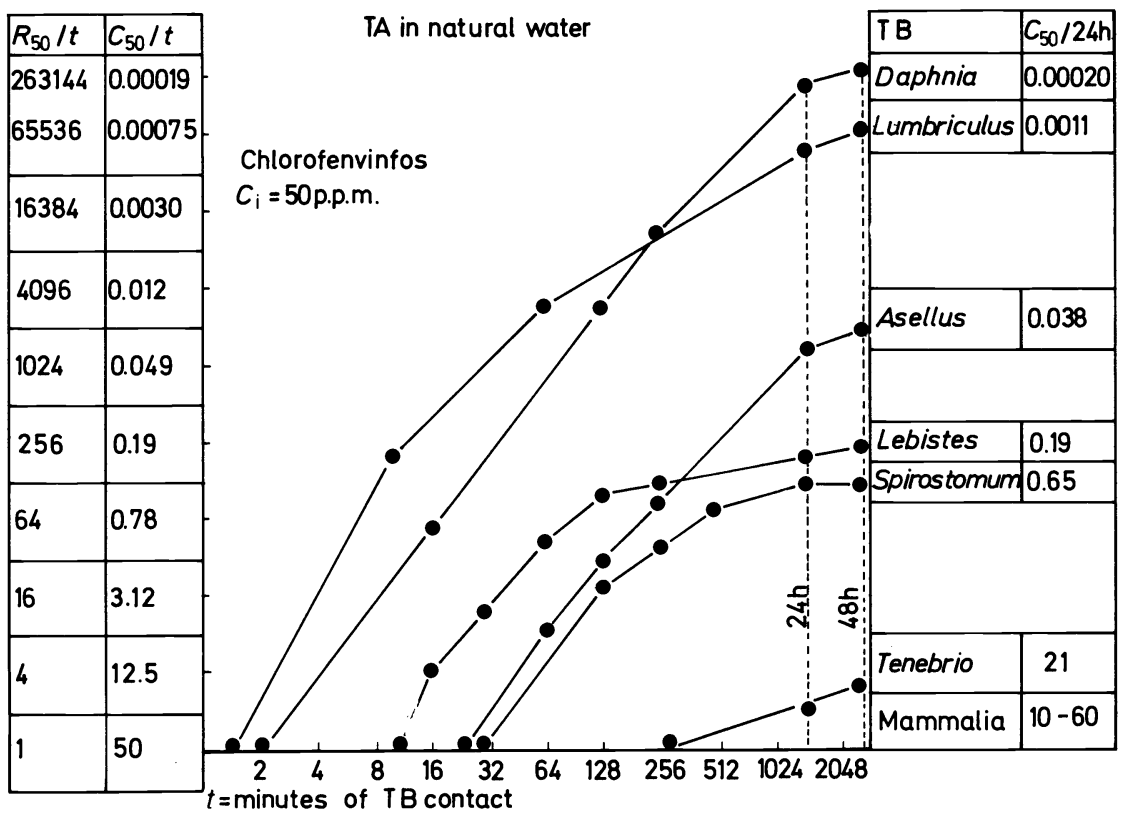

Figure 12. Multitest TA of Chlorofenvinfos, the data analogous to those of Figure 11 
THE USE OF BIOLOGICAL TESTS :OR THE EXAMINATION OF PESTICIDES

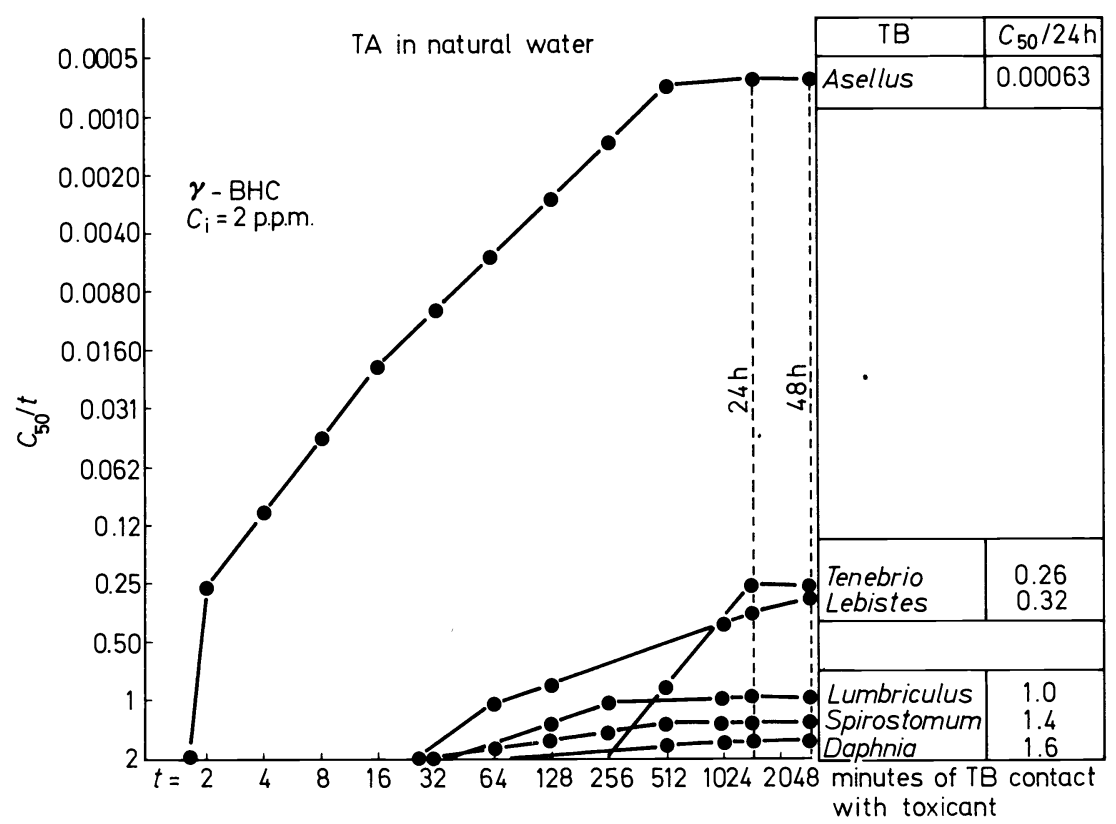

Figure 13. Multitest TA of gamma-BHC, the data analogous to those of Figure 11

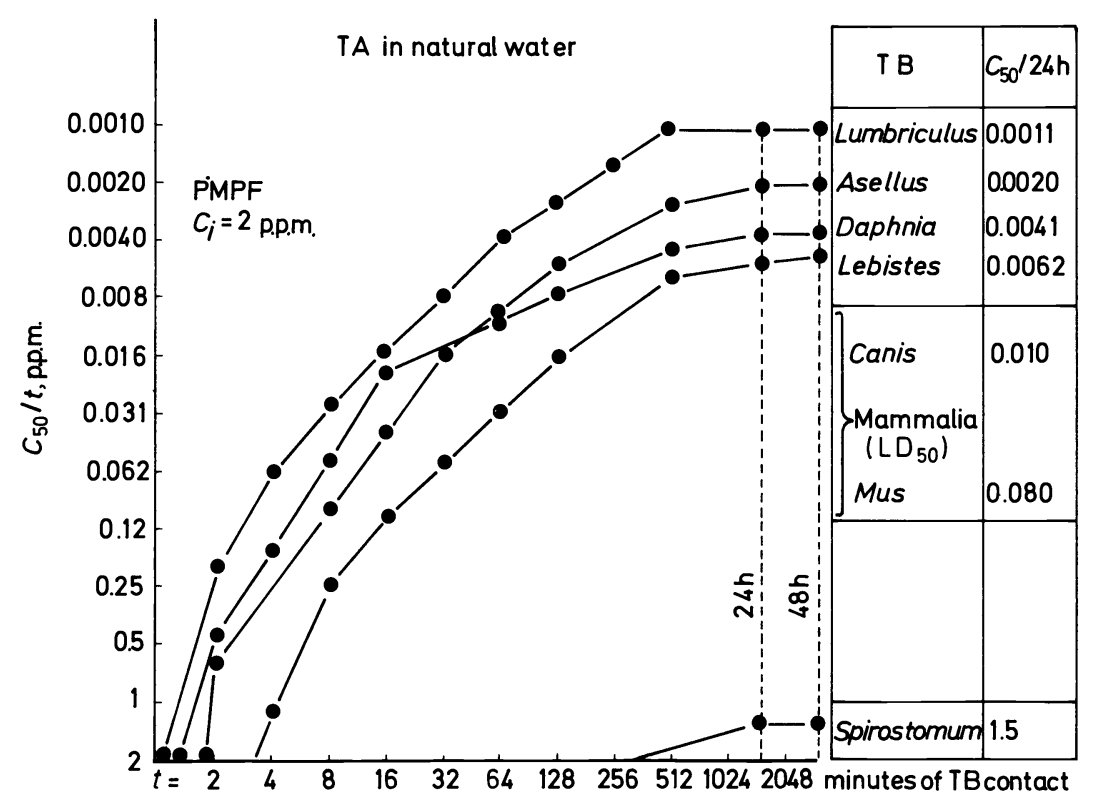

Figure 14. Multitest TA of PMPF, the data analogous to those of Figure 11 
TA method, standards are not necessarily needed, but only detailed records of symptoms and the range of TR.

The course of TR for different toxicants and TB is given in figures. Points in the coordinate system indicate the value of $C_{50}$ recorded at particular times of response $\left(C_{50} / t\right)$. When the materials of unknown concentration are analysed, the value of $R_{50} / t$ is used (Figures 11-15).

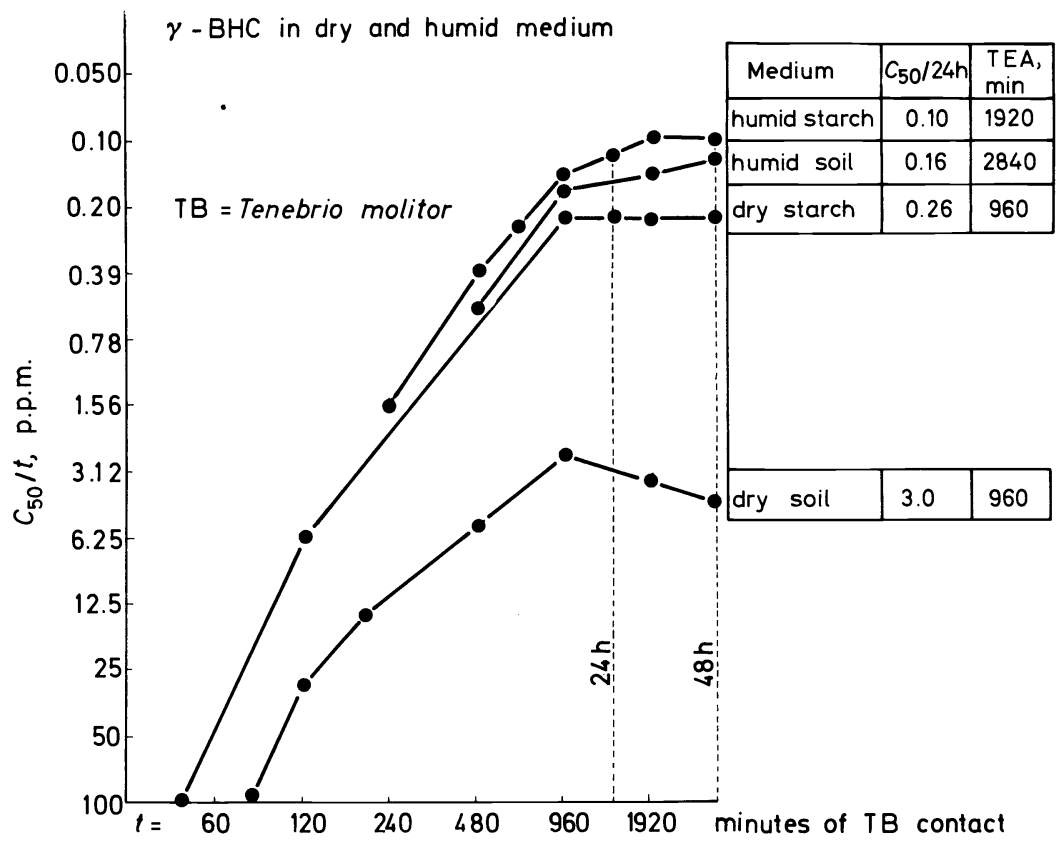

Figure 15. The influence of medium humidity $(20 \%)$ on TR progress in dilution series of BHC (starch impregnation)

\section{(8) Tests of interrupted observation (TIO) and continuous observation (TCO)}

Records of response at the time intervals fixed in advance $(t)$ provide the values of $C_{50} / t, R_{50} / t$ and $t \mathrm{EA}$. A set of many $t$ values increases the basis for statistical analysis of the toxicant and for comparison with the standard. In addition, the values of $C_{50} / t$ or $R_{50} / t$ provide a basis for graphic representation of the results. This method can be defined as a test of interrupted observation (TIO).

The close relation between the onset of TR in TB and toxicant concentration makes it possible to use continuous observation and to note the time of TR in particular TB at each concentration of the toxicant. In this way an average $\bar{t}$ value is obtained for a group of TB composed of 10-30 individuals, depending on the level of the desired accuracy of the analysis. In that case, a sample of the material under study may be used without dilution or it may be diluted to half so that TR could be produced in all TB within 3-60 minutes. The relation between $\bar{t}$ and insecticide concentration is shown in Figure 11 and 
THE USE OF BIOLOGICAL TESTS FOR THE EXAMINATION OF PESTICIDES

Table 3. TA of DDVP steam in dynamic micro-air chamber-washer system (compare Figure 3) TB I = Tenebrio molitor ; TB II = Daphnia magna

Steam source: $0.25 \mathrm{~g}$ of DDVP on $10 \mathrm{~cm}^{2}$ of impregnated Whatman filter-paper

\begin{tabular}{clcccc}
\hline & \multicolumn{2}{c}{ Test response (TR) of TB $(t$ values, min) } & \multicolumn{2}{c}{$\begin{array}{c}\text { Detected concentration } \\
\text { of DDVP (p.p.m.) }\end{array}$} \\
\cline { 2 - 4 } $\begin{array}{c}\text { Duration } \\
\text { of steam } \\
\text { flow-30L/h } \\
(\mathrm{min})\end{array}$ & $\begin{array}{c}\text { In miniature } \\
\text { dynamic air } \\
\text { chamber on } \\
\text { Tenebrio }\end{array}$ & $\begin{array}{c}\text { In Schott } \\
\text { washer I on } \\
\text { Daphnia }\end{array}$ & $\begin{array}{c}\text { In Schott washer } \\
\text { II on Daphnia }\end{array}$ & $\begin{array}{c}\text { Washer I } \\
(100 \mathrm{ml})\end{array}$ & $\begin{array}{c}\text { Washer II } \\
(100 \mathrm{ml})\end{array}$ \\
\hline 1 & without TR & 11.5 & without TR & 0.013 & 0 \\
2 & without TR & 7.10 & without TR & 0.041 & 0 \\
5 & partial TR & 2.22 & without TR & 0.40 & 0 \\
10 & total TR & 1.43 & without TR & 0.82 & 0 \\
15 & total TR & 1.17 & without TR & 0.92 & 0 \\
300 & total TR & $>1.00$ & 35.2 & 2.00 & 0.0070 \\
\hline
\end{tabular}

Table 3. Due to the test of continuous observation. TA can be conducted during a short time and in a small volume of the sample.

\section{(9) Reversibility of TR}

TB removed from the analytical series of a toxicant (pesticide) and put into a pure environment (AEE, TEE) are used for further investigations to determine the degree of reversibility of TR. The highest degree of reversibility of the response to insecticides was found in Lumbriculus (criterion of the specification of toxic substances). Tenebrio is restored after heavy doses of pyrethrins; after a 24 -h period of contact with $\mathrm{BHC}$ it is restored at a concentration of three times that of $C_{50} / 24 h$. The reversibility index $(R v I)$ may be important for the practical evaluation of insecticides. It is calculated for a particular $t$ according to the formula

$$
R v I=C_{\mathrm{r} 50} /\left(C_{50} / t\right)
$$

Table 4. Reversibility of TR in fresh water after one 24-h TB contact with toxicant dilution series

\begin{tabular}{llllllll}
\hline \multirow{2}{*}{ Toxicant } & & \multicolumn{3}{c}{$R v I$ values throughout restitution time } & \multicolumn{2}{c}{ Maximum } \\
& TB species & \multicolumn{3}{c}{ (TR) } \\
\cline { 3 - 7 } & & $\mathrm{RT}=24 \mathrm{~h}$ & $48 \mathrm{~h}$ & $72 \mathrm{~h}$ & $96 \mathrm{~h}$ & $\mathrm{RT}, \mathrm{h}$ & \multirow{2}{*}{$\mathrm{RvI}$} \\
\hline DDVP & Daphnia & 1 & 0.72 & + & + & + & + \\
ChLVF & Lebistes & 0.83 & 0.76 & 0.76 & 0.76 & 24 & 0.83 \\
ChLVF & Tenebrio & 0.88 & 0.76 & 0.53 & 0.53 & 24 & 0.88 \\
DDVP & Lebistes & 1 & 1 & 1 & 1 & 24 & 1 \\
DDVP & Tenebrio & 1.1 & 1.4 & 1.4 & 1.4 & 48 & 1.4 \\
$\gamma$-BHC & Tenebrio & 2.1 & 3.2 & 3.2 & 3.2 & 48 & 3.2 \\
$\gamma$-BHC & Lumbriculus & 1.6 & 3.5 & 5.3 & 7.6 & 254 & 10 \\
ChLVF & Lumbriculus & 2.0 & 3.7 & 17 & 32 & 336 & 103 \\
DDVP & Lumbriculus & 7.4 & 13 & 54 & 123 & 96 & 394 \\
\hline
\end{tabular}

Abbreviations: $\mathrm{RT}=$ duration of restitution; $R v I=\left(C_{50} / \mathrm{RT}\right) /\left(C_{50} / 24 \mathrm{~h}\right) ;+$ denotes lethal reaction. 


\section{ALFRED KAMIŃSKI}

where $C_{\mathrm{r} 50}$ is the concentration in the analytical series to which TR was shifted for a TB absorbing a toxicant during time $t$, and $C_{50} / t$ is the threshold concentration for TR in TB, determined during time $t$ of absorption (Table 4).

\section{(10) Qualitative analysis and evaluation using TA}

Developed TA is primarily used to characterize toxicodynamic properties of pesticides. Conducted on numerous TB, including the determination of the effects on their reproduction, it provides a part of the ecological screening. This part gives a taxonomic spectrum of toxic activity (BSTA) which is specific for each poison. On the other hand, the quantitative criteria, symptomatology of TR, BSTA, $t \mathrm{EA}$ and $R v I$ are the basis for the identification of insecticides by TA and simultaneous quantitative analysis.

When the material is contaminated with a mixture of toxicants, objects with a low sensitivity to insecticides are used (Spirostomum) to detect nonpesticide toxicants, such as heavy metals and detergents, because in that case they perform the role of STB. In further analyses particular insecticide STB are included in the TA. Preparatory elimination (without reactors)--thermal, distillation and sorptive - has an auxiliary role, the details of which cannot be discussed here.

If a sample contaminated with a toxicant of unknown quality and quantity is the subject of TA, only the detailed symptomatology of the response of the TB system and the ease of determining the value of $R_{50}$ for a particular TB can be found. This is a multitest analysis of the cumulative toxicity (MACT) of industrial sewage, river, soil, atmospheric fallout etc., independent of the amount and chemical quality of poisons contributing to the total toxicity. MACT should be a component of the chemical indication of environmental pollution and the creation of a sanitary-ecological security margin, besides that of CPA.

The studied TB are frequently many times more sensitive to the tested toxicants than the human organism. So, this security margin is quite correct from the point of view of protecting nature. MACT provides a basic criterion for the estimation of the effects of soil and water pollution with pesticides.

The CPA method of qualitative analysis of each sample of material contaminated with pesticides provides the same number of criteria to specify pesticides as TA does. But when the concentration of toxicants is low and the size of samples is small, the latter is preferred. When a mixture of toxicants is studied, a system of the MACT indices on any number of TB can always be found.

\section{(11) The use of TA for the detection of toxicological interactions (TI)}

Interactions among different toxicants (synergism, addition, antagonism) are the subject of many investigations ${ }^{18}$. Commonly used methods of TI determination are rather complex. In our experiments the TI screening used was based on the toxicological parameters of the ratio of the concentration of particular components of the mixture. A constant $0.5 C_{50} / 24 \mathrm{~h}$ of one toxicant is used in the presence of the whole gradient of concentrations of another toxicant and vice versa. Synergism occurs when the effect of the mixture on TB is intensified. In that case the threshold TR is shifted towards lower concentrations. When antagonistic interaction takes place, e.g. in the presence 
of an antidote, the threshold (limit) is shifted towards the beginning of the gradient. The ratio of weights for both the components of the mixture ranges from a fraction to a multiple. Due to the double versions of weight ratios, an optimum for maximum interaction can be found. In the third version the original mixture of known composition is diluted in such a way that a constant ratio between the weights of both components is preserved. This ratio may be obtained either on the basis of absolute weights (in which case it is not toxicologically defined) or on the basis of the weights proportionally related to the value of $C_{50}$ for each of the components, which provides a better situation for the proper interpretation of the results.

\section{(1) Objectives}

\section{ECOLOGICAL TEST ANALYSIS (ETA)}

This analysis, like TA, has two objectives: first, an assessment of the response of the biont, i.e. in this case a whole natural model ecosystem (ETS) or a part of it, to contamination with a toxicant (pesticides), and secondly, the fate of the toxicant in contact with the components of the ETS.

These two problems are equally important from the point of view of the practical application of pesticides. The complex objective of ETA may be divided into smaller units by the use of laboratory ETS. Methodologically the following phenomena can be distinguished here:

(1) contamination and its effects on the structure and functioning of ETS,

(2) detoxification by biomass, natural absorptive systems and by chemical processes in ETS,

(3) threshold of tolerance to contamination with toxicant,

(4) restoration after a single contamination,

(5) the above-mentioned phenomena under the dynamic conditions of a continuous flow of the toxicant through ETS.

\section{(2) Aquatic ETS-static}

During the long-term experiments a static biological unit was devised, which formed an element of the multi-component battery of ETS (Figure 16). This unit (4 litres) consists of all ecological trophic levels of the natural waters of alpha-mesotrophic type: sand-a layer of $4 \mathrm{~cm}$, aquatic plants such as Valissneria spiralis, Myriophyllum pinnatum, and aquatic TB such as Asellus, Lumbriculus, Planorbarius, Spirostomum and Daphnia. At controlled photoperiod and uniform nutrition (dried leaves of Populus nigra and Populus canadensis) this ETS can be maintained in a state of functional balance (homeostasis) over many months, independent of the season. The biomass of plants, animals, microplankton, microbenthos and saprobionts are able to maintain a proper nutrient cycle. The chemical properties of the water are normal. Due to the mineralization processes a sedimentary material similar to young peat is deposited without detectable decay processes. The state of homeostasis is identified on the basis of the behaviour of animal TB sensitive to oxygen deficiency and the changes in the chemical properties of the water.

The battery of ETS plays the same role as TB in TA. TR resulting from pollution with toxicants can be observed in all bionts (plants and animals) and 


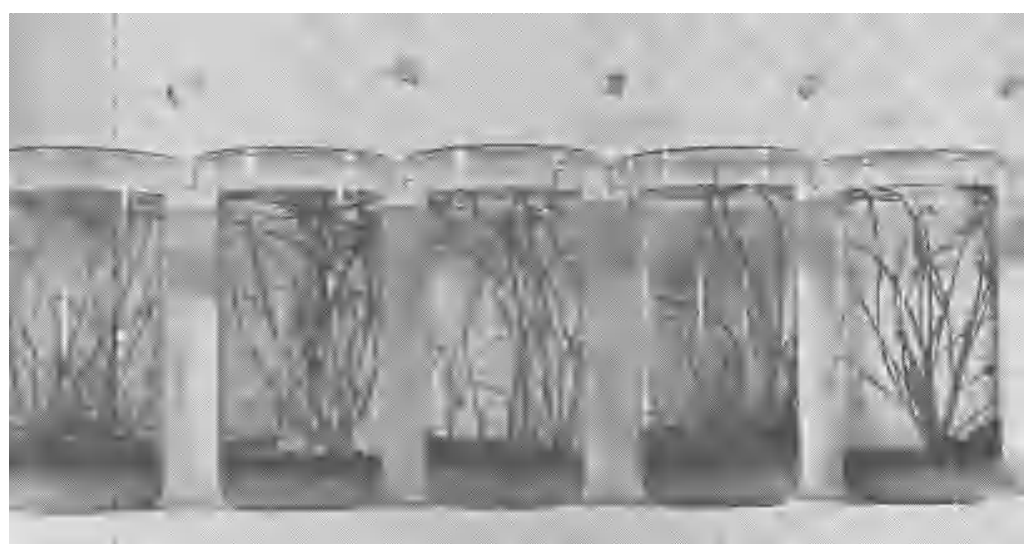

Figure 16. A battery of 5 aquatic ETS-units (vessels)

in the processes of energy flow, which can be indirectly measured (chemical properties of water, oxygen requirements, the rate of food mineralization, increase in biomass). Static ETS are used to examine the ecological results of a single contamination with a pesticide or other toxicant at different doses. From many problems concerned with ETA only several selected examples can be presented here.

\section{(3) Detoxification capacity of ETS}

A polluted unit of ETS is detoxified gradually in a time proportional to the concentration and specific persistence of the toxicant. Samples of the water are examined by TA on STB. The results are presented in the table (Table 5) and figures (Figure 17). By replication of the treatment after complete detoxification it is possible to determine the detoxification capacity of the ETS, which generally decreases as the number of treatments increases.

The experiments indicate that $\gamma$-BHC and Chlorofenvinphos are extremely persistent toxicants. They react with a minute amount of the receptor and are involved neither in the metabolic processes nor in the chemical processes of the ETS. DDVP is degraded by ETS in a much shorter time. Mercuric chloride reacts with a large amount of receptor and with the total absorption and chemical potential of the ETS. It disappears rapidly. When the treat-

Table 5. The maximum values of toxicant concentration for a complete detoxification of water $(\mathrm{DC} / \mathrm{T})$ by aquatic ETS - static (4 litres) in $\mathrm{T}=24 \mathrm{~h}$

Initial biomass fresh weight: plants $40 \mathrm{~g}$, animals $2 \mathrm{~g}$, standard detritus $40 \mathrm{~g}$. Permanent aeration (15 litres of air in $1 \mathrm{~h}$ ), temper. $19^{\circ} \mathrm{C}$.

\begin{tabular}{|c|c|c|c|c|c|}
\hline Toxicant & $\mathrm{HgCl}_{2}$ & PMPF & DDVP & ChLVF & $\gamma-\mathrm{BHC}$ \\
\hline DC/24 h (p.p.m.) & 1.2 & 0.50 & 0.053 & 0.0047 & 0.0041 \\
\hline $\begin{array}{l}\text { Detectability by } \\
\text { TA (p.p.m.) } \\
\text { TB-species }\end{array}$ & $\begin{array}{c}0.040 \\
\text { Spirostomum }\end{array}$ & $\begin{array}{c}0.0013 \\
\text { Lumbriculus }\end{array}$ & $\begin{array}{c}0.0010 \\
\text { Daphnia }\end{array}$ & $\begin{array}{l}0.00040 \\
\text { Daphnia }\end{array}$ & $\begin{array}{r}0.00063 \\
\text { Asellus }\end{array}$ \\
\hline
\end{tabular}


Detoxication of water in static ETS

-residues of initial charge

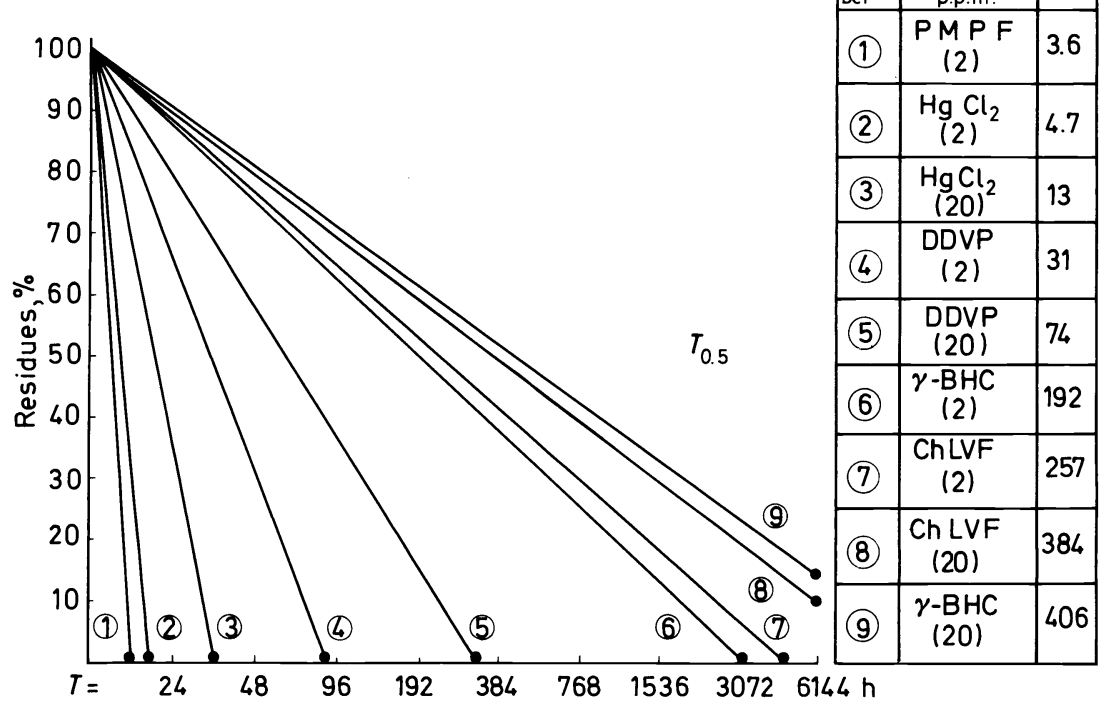

Figure 17. The decrease of active toxicant residues in aquatic ETS with $T_{0.5}$ values

ments with toxicants are replicated and the receptors are saturated, the detoxification rate rapidly decreases. The effect of detoxification is best characterized by $T_{0.5}$, i.e. the time to half-degradation. Also the maximum values of concentration for a complete detoxification by ETS in $24 \mathrm{~h}$ (Table 5) provides good information. They are the highest for PMPF and the lowest for Chlorofenvinphos and $\gamma-\mathrm{BCH}$.

\section{(4) Aquatic ETS-dynamic}

The battery of ETS united in a flow system of communicating vessels (Figure 18) is subjected to permanent pollution. Its role for the analytical ecology of contamination corresponds to the role of static systems. In addition, its main objective is to carry out a complete detoxification by means of a definite number of linked units. The efficiency of the battery, e.g. composed of five units, depends on the input of a toxicant. The required value is the maximum rate of inflow (in $\mathrm{ml} / \mathrm{h}$ ) ensuring detoxification along the whole battery. The criterion of detoxification is the complete survival of STB in the last unit and the results of quantitative analyses in the remaining units (Table 6).

To start the experiment, a solution of the toxicant of known concentrations is poured into the battery. Immediately before the start, unit I is filled with undiluted toxicant. The inflow of toxicant from the source pushes the toxicant already present in unit I into unit II and all successive units. One of the main purposes of the use of the battery is to determine the tolerance to permanent contamination. In that case samples from particular units should be taken every day and the content of toxicant determined on STB. Also the response 


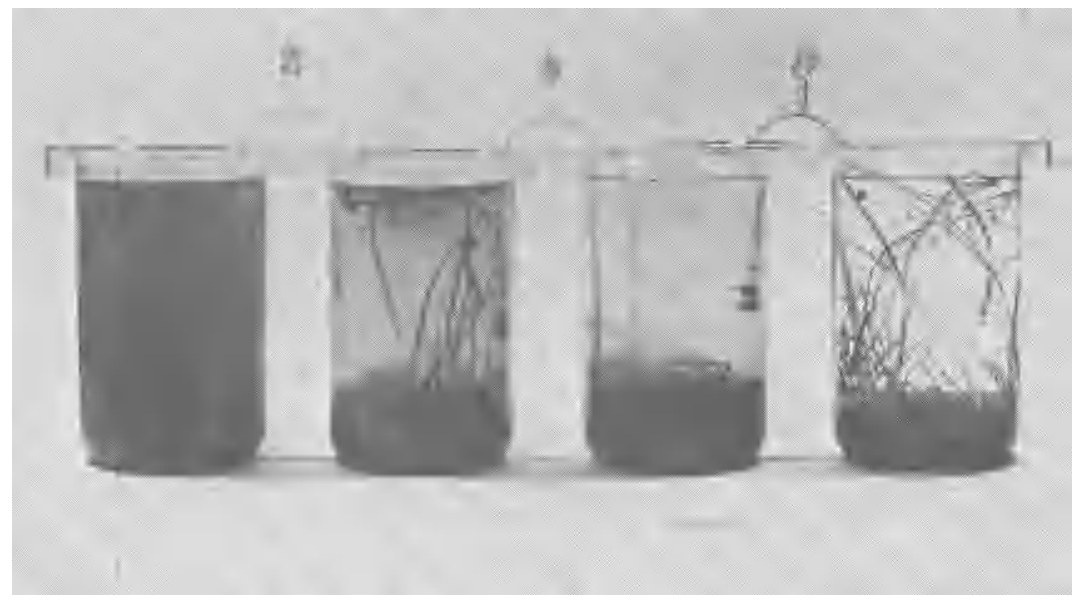

Figure 18. A battery of 4 aquatic ETS-units (communicating vessels) as permanent flow system of toxicant. The first three vessels ( $L$ to $R$ ) show visible TR symptons (ecological catastrophe) after PMPF application, the fourth has no TR

of bionts in situ is determined, with particular reference to the processes of ecological succession. One of the more interesting phenomena is the response of chloroplasts to mercuric chloride, and others are the rapid succession of anaerobic organisms in the presence of PMPF, and also significant values of the persistent pesticides (BHC and Chlorofenvinphos) as reagents determining the hydrodynamic parameters of ETS functioning. They play the role of stable tracers of the poured fluid and are determined on STB above 0.0003 p.p.m. Their role is similar to that of radioactive tracers.

Table 6. TA of $\mathrm{HgCl}_{2}$ in dynamic ETS battery (Five communicating vessels, each 4 litres of capacity).

Permanent toxicant inflow into vessel I: $40 \mathrm{mg} / 24 \mathrm{~h}$ in 8 litres of water $\mathrm{TB}=$ Spirostomum ambiguum; Temperature $19^{\circ} \mathrm{C}$

\begin{tabular}{cccccc}
\hline \multirow{2}{*}{$\begin{array}{c}\text { Duration } \\
\text { of toxicant } \\
\text { inflow, h }\end{array}$} & \multicolumn{4}{c}{ Detected concentration of active toxicant in water, p.p.m. } \\
\cline { 2 - 6 } & Vessel I & Vessel II & Vessel III & Vessel IV & Vessel V \\
\hline 0 & 5 & 0 & 0 & 0 & 0 \\
24 & 1.10 & 0 & 0 & 0 & 0 \\
48 & 1.25 & 0 & 0 & 0 & 0 \\
96 & 130 & 0.15 & 0 & 0 & 0 \\
192 & 1.25 & 0.31 & 0 & 0 & 0 \\
384 & 2.42 & 0.63 & 0.057 & 0 & 0 \\
480 & 2.61 & 0.67 & 0.062 & 0 & 0 \\
\hline
\end{tabular}

\section{(5) Terrestrial ETS}

Terrestrial models of test systems have different sizes and functions. They work in a similar way to aquatic ETS. They represent fragments of forests, 


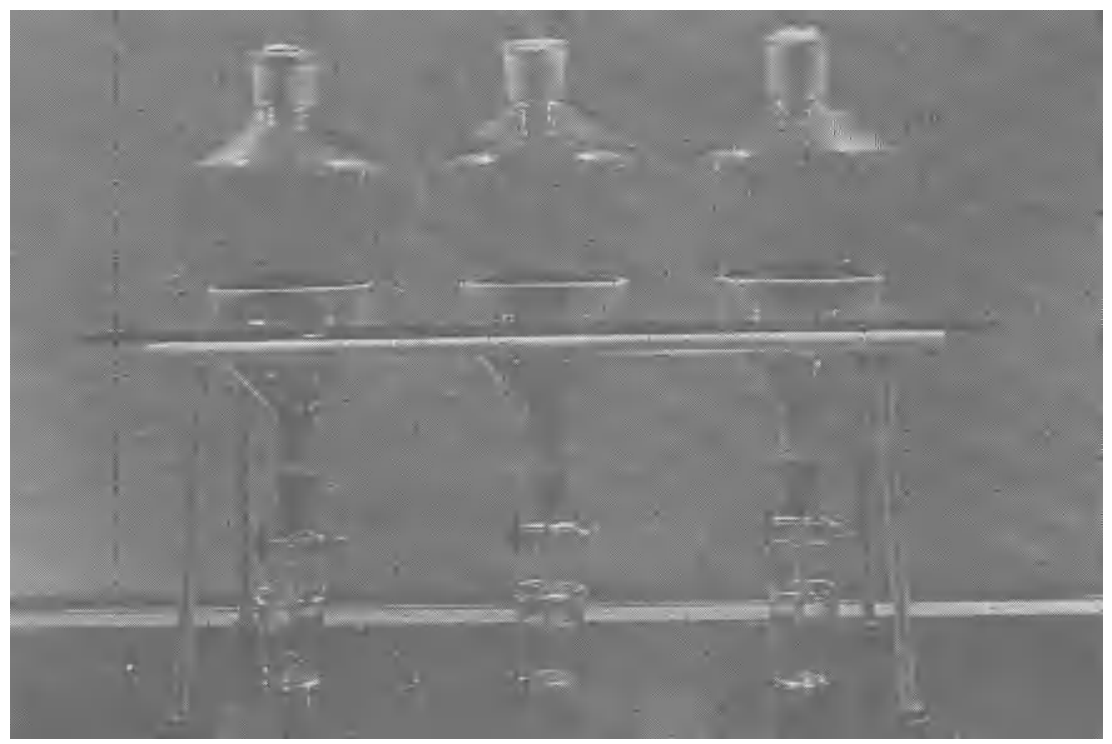

Figure 19. A small terrestrial ETS battery for environmental TA of pesticides in soil and in extracts

meadows, riverside environments, crop fields, municipal areas. Static ETS used for one experiment are small (Figure 19). They contain only soil or a fragment of plant cover. They function like lysimeters with hemispherical glass cover and with controlled ventilation. By their use whole blocks of soil and selected parts of the system can be analysed (Figure 20).

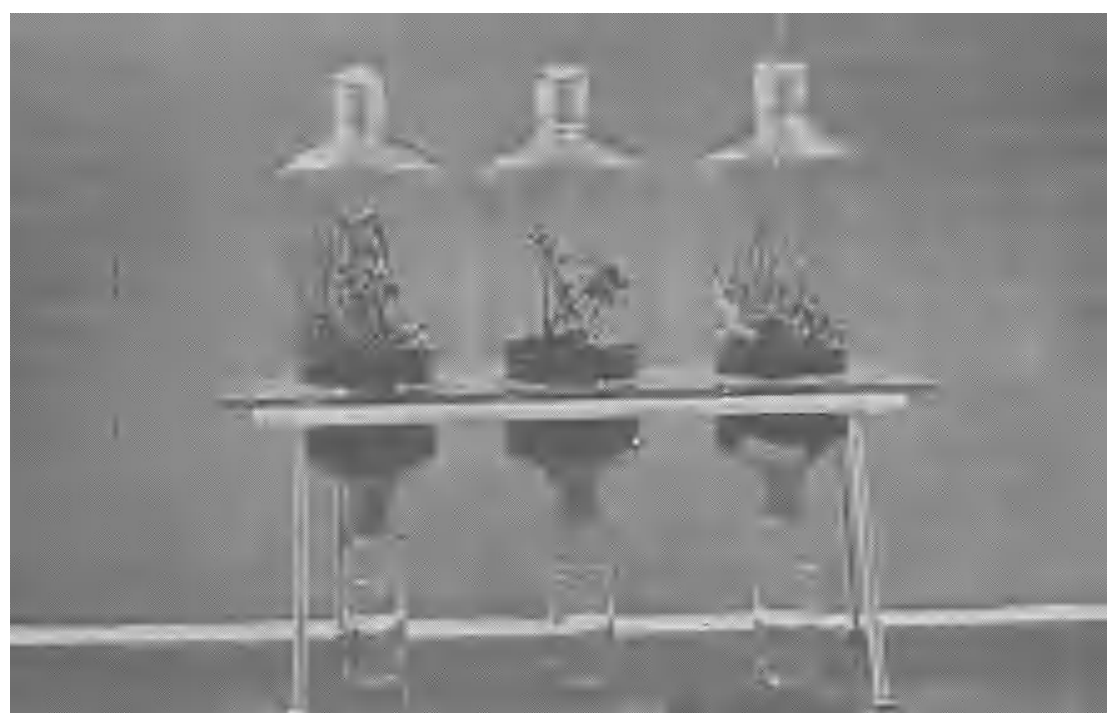

Figure 20. A small terrestrial ETS battery for ecological screening of pesticides 


\section{ALFRED KAMIŃSKI}

ETS of medium size (Figure 21) are provided with an installation for the flow of gas and they are also bioindicators for volatile forms of toxicants (Figure 22).

Large ETS $\left(1 \mathrm{~m}^{2}\right)$ represent fairly complete models of agrocenoses, forest and other biocenoses. To extract pesticides, all the soil or samples of soil

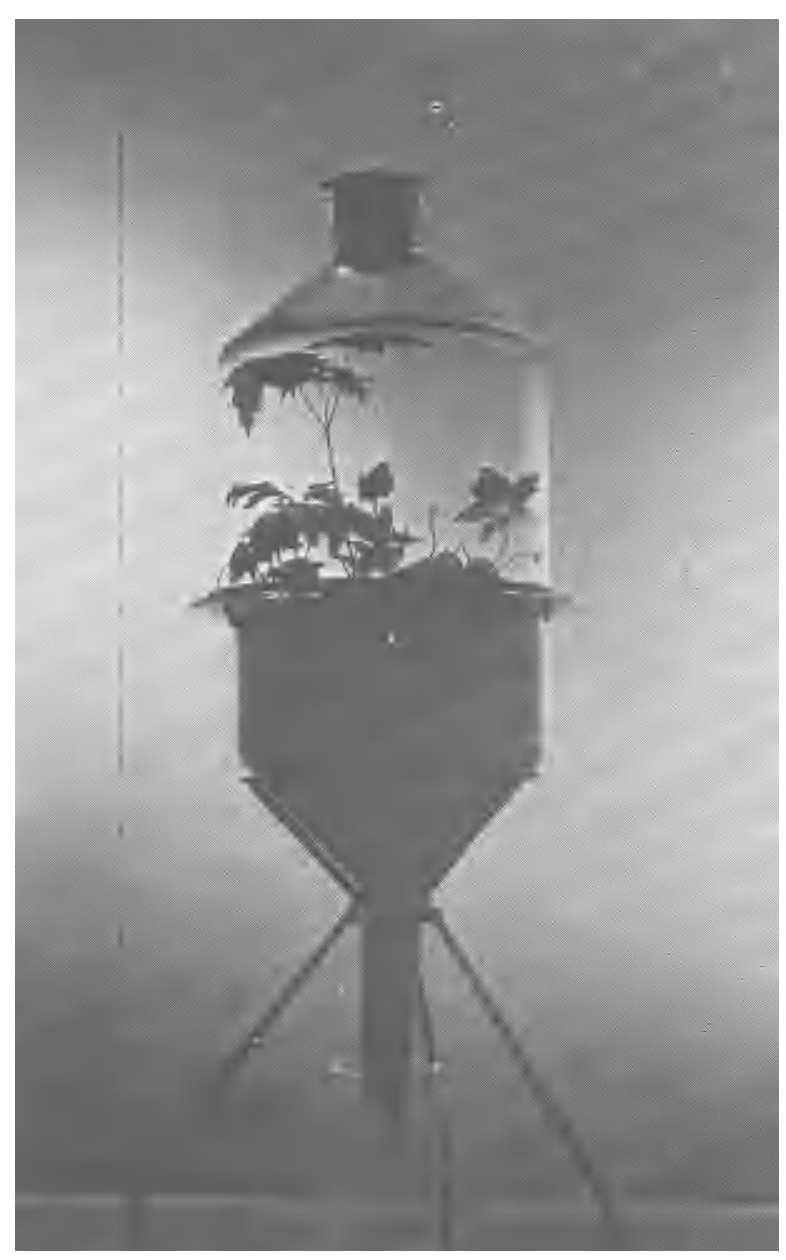

Figure 21. A terrestrial ETS of medium size for long time experiments and TA of ecosystem fragments or total extracts

from large ETS are treated with a solvent (in a Buchner's funnel). The largest ETS are used to study the cumulative impact of chemicals. They are characterized by a diversified structure of plant species composition and a large potential of homeostasis (Figure 23). In addition, the detoxification activity of these ETS is estimated in relation to different pesticides, as in aquatic systems. The role of TA in these analyses is of basic importance. Due to this 


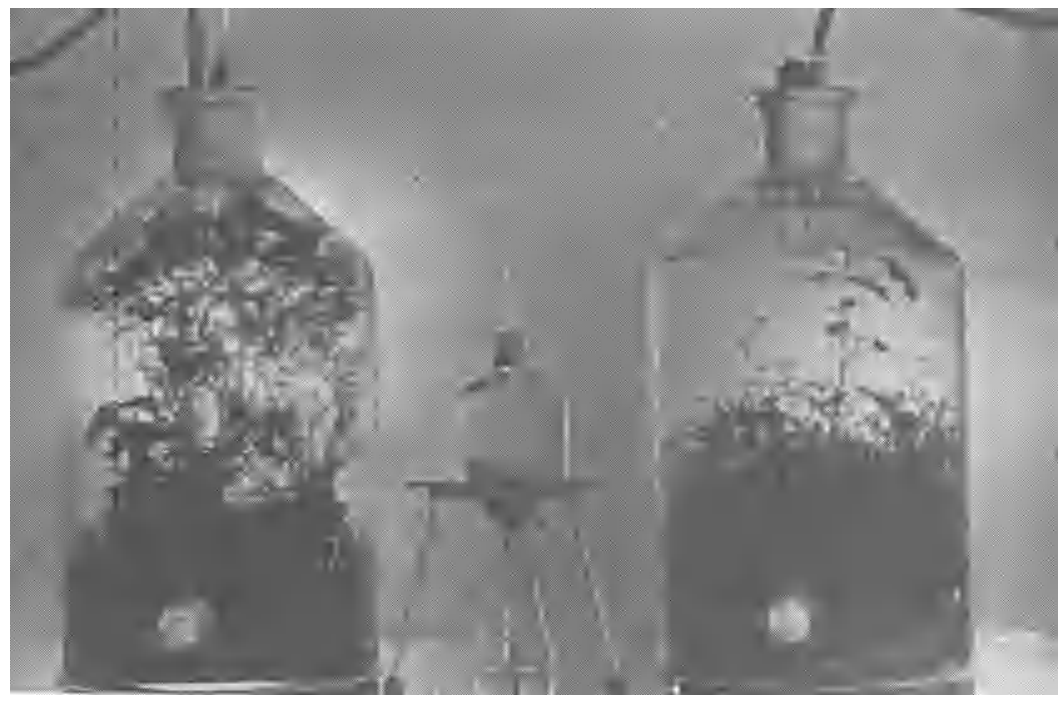

Figure 22. Terrestrial ETS of medium size for permanent toxicant flow (steam or fluid form) and one small ETS-vessel for comparison of sizes

technique it is possible to carry out experiments in situ, directly on the surface of soil treated with water (Asellus and Lumbriculus) or in extracts from different structures of $\operatorname{ETS}^{16,19,20}$.

Terrestrial ETS provide an experimental basis for planning indicatory biocenoses in open areas and also biocenoses tolerant to present treatments with chemicals.

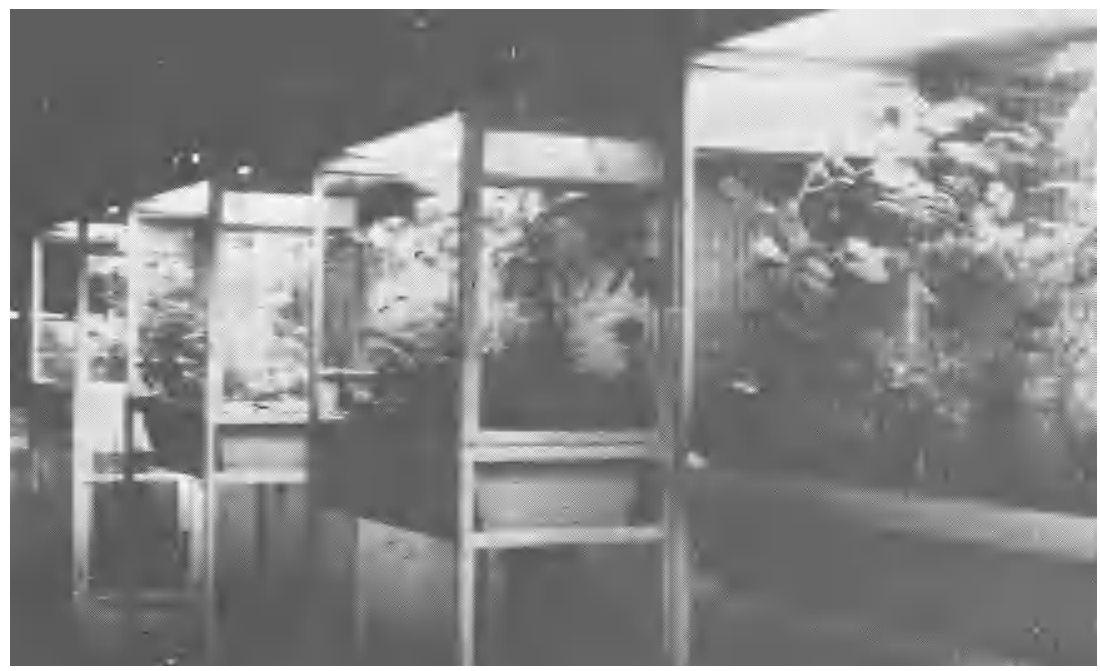

Figure 23. A battery of large ETS for semi-field experiments with pesticides and other toxicants 


\section{(6) Inversion of the ETA function}

Readily metabolized IChE, such as PMPF, $\mathrm{HgCl}_{2}$, DDVP and Dipterex are used for the detection of living matter in different materials of a complex structure, such as soil or sediments. Comparison of the effect of detoxification in natural and denatured materials characterizes the content and activity of a biotic factor. The value of such a reagent increases with an increase both in its influence upon the environment and the rate at which it is metabolized by the biomass. At low concentrations of $0.1-1$ p.p.m. its toxicity rapidly disappears due to the metabolic activity of small biomass (small plants and animals). The degree of disappearance is proportional to the amount of active biomass. The reactive system is composed of PMPF-Asellus, or DDVPDaphnia, or $\mathrm{HgCl}_{2}-$ Daphnia and Spirostomum.

The concentration of DDVP drops from 1 p.p.m. to 0.25 p.p.m. after 30 min of incubation with natural humus soil, and to 0.120 p.p.m. when it is incubated with denatured soil. So, the index of metabolic detoxification activity (MDA) is 4.8 , the absorption of dead soil being taken as 1 . The analysis with an accuracy of 0.01 p.p.m. of detoxification effects after the incubation of a reagent with soil takes only a few minutes (compare Table 7).

Table 7. Biodegradation and absorption of toxicants by organic detritus from an eutrophic lake. $\mathrm{T}=24 \mathrm{~h}$, temperature $19^{\circ} \mathrm{C}$, permanent aeration

\begin{tabular}{|c|c|c|c|c|c|c|}
\hline \multirow{2}{*}{ Toxicant } & \multirow{2}{*}{$M(\mathrm{~g})$} & \multirow{2}{*}{$C_{\mathrm{i} M}$ (p.p.m.) } & \multirow{2}{*}{$C_{\mathrm{i}}$ (p.p.m.) } & \multicolumn{2}{|c|}{$\begin{array}{l}\text { Final concentration of } \\
\text { toxicant }\left(C_{\mathrm{f}}\right) \text { in p.p.m. }\end{array}$} & \multirow{2}{*}{$\begin{array}{l}\text { Index of } \\
\text { metabolic } \\
\text { detoxification } \\
\text { (MDI) }\end{array}$} \\
\hline & & & & $\begin{array}{c}\text { sterilized } \\
\text { detritus } C_{\mathrm{f}_{1}}\end{array}$ & $\begin{array}{l}\text { living } \\
\text { detritus } C_{\mathrm{f}_{2}}\end{array}$ & \\
\hline DDVP & 20 & 5 & 1 & 0.20 & 0.021 & 9.5 \\
\hline $\mathrm{HgCl}_{2}$ & 1 & 1000 & 10 & 0.21 & 0.054 & 4.0 \\
\hline PMPF & 20 & 5 & 1 & 0.0085 & 0.0025 & 3.4 \\
\hline ChLVF & 20 & 5 & 1 & 0.46 & 0.32 & 1.4 \\
\hline$\gamma$-BHC & 20 & 5 & 1 & 0.21 & 0.19 & 1.1 \\
\hline
\end{tabular}

Abbreviations: $M$ is fresh weight of detritus in $100 \mathrm{ml}$ of water; $C_{\mathrm{iM}}$ is charge of toxicant per $1 \mathrm{~g}$ of detritus; $C_{\mathrm{i}}$ is initial concentration of toxicant in the water; $C_{\mathrm{f}}$ is final residue of active toxicant in the water, and MDI $=C_{\mathrm{f}_{1}} / C_{\mathrm{f}_{2}}$

Quantitative analysis of metabolic activity in relation to the above-mentioned toxicants is based on an analytical series of decreasing amounts of soil, sediment and tissue incubated with a toxicant at a constant concentration. Complete detoxification at the beginning of the series is the maximum limit, detoxification by half is the medium limit and a lack of detoxification is the minimum limit. These limits are modified by the value of $C_{\mathrm{i}} /$ orginal concentration of the toxicant (reagent).

This information would be useless here if there was no need for the prediction of pesticide doses for crop protection. It is also needed to determine the effect of additional treatments with chemicals on the detoxification activity of different soils, and to characterize the biotic potential of the environment on the basis of the index of detoxification capacity (MDA), besides enzymatic characteristics. 


\section{SUMMARY}

The TA and ETA techniques presented here are in the first stage of development. The basic purpose is to determine the environmental-toxicological characteristics of all toxicants polluting the natural environment, with particular emphasis on pesticides.

The enlarged TA and ETA should increase the range of information on pesticides and other toxicants in the following directions:

(1) Detectability in different materials and concentrations difficult to study with other methods,

(2) Effects of pesticides on the structure and functioning of natural aquatic and terrestrial ecosystems,

(3) Effect of the natural environment on the biological activity and persistence of pesticides,

(4) Stimulation of progress in the production of new pesticides less damaging to biocenoses (with optimum BSTA),

(5) Development of a bioindicatory security margin which can regulate the quality and intensity of treatments with pesticides,

(6) Current ecological estimation of the cumulative impact of chemicals on the natural environment,

(7) Ecological evaluation of new pesticides,

(8) Utilization of readily metabolized toxicants and STB as reagents characterizing the biomass of environments without disturbing their natural structures,

(9) Planning ecosystems more resistant to the necessary treatments with chemicals,

(10) Adaptation of the most sensitive natural ecosystems for performing the role of bioindicators both in the laboratory and in the field.

Increasing pollution of the environment produces a situation in which STB are protected in laboratories and their natural habitats are deprived of them. This situation can be improved by the application of new methods for the chemical control of pests (repellants, attractants, hormones, chemosterilants) and by lowering the total impact of chemicals. In that case STB will return to their natural habitats as indicators of their purity and the role of STB will be performed only by selectively sensitive pests. At the moment they should gradually pass to the higher levels of specific sensitivity under the influence of new pesticides. It should be expected that TA and ETA will be still more effectively used for monitoring these processes.

\section{INDEX OF ABBREVIATIONS}

$\begin{array}{ll}\text { AEE } & \text { aquatic experimental environment } \\ \text { BCA } & \text { biochemical analysis } \\ \text { BSTA } & \text { taxonomic spectrum of toxic activity } \\ \text { C } & \text { concentration } \\ \text { CPA } & \text { chemicophysical analysis } \\ \text { EE } & \text { experimental environment } \\ \text { EPH } & \text { energy potential of homeostasis } \\ \text { ETA } & \text { ecological test analysis }\end{array}$




\section{ALFRED KAMIŃSKI}

$\begin{array}{ll}\text { ETS } & \text { ecological test system } \\ \text { IChE } & \text { inhibitor of choline esterase } \\ \text { LC } & \text { lethal concentration } \\ \text { LR } & \text { lethal response } \\ \text { MACT } & \text { multitest analysis of cumulative toxicity } \\ \text { MDA } & \text { metabolic detoxification activity } \\ \text { NE } & \text { natural environment } \\ R & \text { dilution } \\ R v I & \text { reversibility index } \\ \text { SPB } & \text { system of permanent bioindication } \\ \text { ST } & \text { standard toxicant } \\ \text { STB } & \text { sensitive test biont } \\ t & \text { time } \\ \text { TA } & \text { test analysis } \\ \text { TB } & \text { test biont } \\ \text { TDC } & \text { toxic-dynamic characteristic } \\ t \text { EA } & \text { critical time-moment of effective absorption } \\ \text { TEE } & \text { terrestrial experimental environment } \\ \text { TI } & \text { toxicological interaction }\end{array}$

\section{REFERENCES}

1 J. R. Busvine, A Critical Review of the Techniques for Testing Insecticides, London (1957).

2 R. L. Metcalf, Organic Insecticides, New York-London (1955).

3 W. Eichler (ed.), Handbuch der Insektizidkunde, Berlin (1965).

${ }^{4}$ G. L. Ellman, Biochem. Pharmacol. 7, 88 (1961).

5 T. Namba, Bull. Wld Hlth. Org. 44, 289 (1971).

${ }^{6}$ F. Hauschild, 'Pharmakologische Wirkungen der Insektizide' in: W. Eichler (ed.), Handbuch der Insektizidkunde, pp 433-452. Berlin (1965).

7 S. S. Lee, R. D. Wanchoppe, H. Rizwanul and S. C. Fang, Pesticides Biochem. Physiol. 3, 225 (1973).

8 A. Voronkin and Y. Loshakov, Exper. Vodnaja Toxikologia 5, 168 (1973).

9 J. G. Needham, F. E. Lutz, P. S. Welch and P. S. Galtsoff, Culture Methods for Invertebrate Animals, Dover: New York (1959).

10 G. Meister, Z. Tropenmed. Parasitol. 13, 220 (1962).

11 H. B. N. Hynes, The Biology of Polluted Water, University Press: Liverpool (1960).

${ }^{12}$ V. Sladecek, 'System of water quality from the biological point of view', Arch. Hydrobiol. Suppl. Ergebnisse der Limnologie No. 7. E. Schweizerbart: Stuttgart (1973).

13 W. Bratkowska, Biul. WAM, 16, 49 (1973).

14 A. Kamiński, Angew. Parasitol. 2, 86, 110 (1961).

15 D. J. Finney, Statistical Methods in Bioassay, Griffin: London (1952).

16 A. Kamiński, B. Zawadzki and T. Szymańska, Roczn. Nauk Roln. 95-A-4, 589 (1969).

17 A. Kamiński and T. Kisieliński, Roczn. WIHE 2(5), 211 (1961).

18 Seiroku Sakai, Insect Toxicological Studies on the Joint Toxic Action of Insecticides. Yashima Chemical Industries: Tokyo (1960) (with 573 references).

19 A. Kamiński, Verch. Internat. Verein. Limnol. 16, 969 (1966).

20 R. E. Warner, Bull. World Health Org. 36, 181 (1967). 